\title{
1 Research gaps and new insights in the intriguing evolution of Drosophila seminal proteins
}

4 Hurtado $\mathrm{J}^{*}, 1,2$, Almeida $\mathrm{FC}^{1,2}$, Belliard $\mathrm{SA}^{3}$, Revale $\mathrm{S}^{4}$ and Hasson $\mathrm{E}^{1,2}$

${ }^{1}$ Departamento de Ecología, Genética y Evolución, Facultad de Ciencias Exactas y Naturales, Universidad de Buenos Aires (UBA), CABA, Argentina.

${ }^{2}$ Instituto de Ecología, Genética y Evolución de Buenos Aires, Consejo Nacional de Investigaciones Científicas y

9 Técnicas (CONICET), CABA, Argentina.

${ }^{3}$ Laboratorio de Insectos de Importancia Agronómica, IGEAF (INTA), GV-IABIMO (CONICET), Hurlingham, Buenos

11 Aires, Argentina.

$12{ }^{4}$ Wellcome Trust Centre for Human Genetics, University of Oxford, Oxford, OX3 7BN, UK.

*Author for Correspondence: Juan Hurtado, jhurtado@ege.fcen.uba.ar

\section{Abstract}

While the striking effects that seminal fluid proteins (SFPs) exert on females are fairly conserved among Diptera, they exhibit remarkable evolutionary lability. Consequently, most SFPs lack detectable homologs among the repertoire of SFPs of phylogenetically distant species. How such a rapidly changing proteome "manages" to conserve functions across taxa is a fascinating question. However, this and other pivotal aspects of SFPs' evolution remain elusive because discoveries on these proteins have been mainly restricted to the model $D$. melanogaster. Here, we provide an overview of the current knowledge on the inter-specific divergence of Drosophila SFPs and compile the increasing amount of relevant genomic information from multiple species.

24 Capitalizing the accumulated knowledge in D. melanogaster, we present novel sets of high25 confidence SFP candidates and transcription factors presumptively involved in regulating the expression of SFPs. We also address open questions by performing comparative genomic analyses that failed to support the existence of conserved SFPs shared by most dipterans and indicated that gene co-option is the most frequent mechanism accounting for the origin of Drosophila SFP-coding genes. We hope our update establishes a starting point to integrate, as more species are assayed for SFPs, further data and thus, to widen the understanding of the intricate evolution of these proteins. 


\section{Keywords}

Accessory glands; De novo gene evolution; Gene co-option; Gene turnover; Gene origin; Seminal

35 fluid

\section{Introduction}

During mating, spermatozoa expelled from the testes travel throughout the ejaculatory duct into the female reproductive tract accompanied by a rich repertoire of proteins and peptides known as Seminal Fluid Proteins (SFPs) (reviewed in, e.g., Avila et al. 2011; Avila et al. 2016; Chapman 2008; Ramm 2020). These proteins, likely adapted to sperm competition and fertilization, have been highly studied in Drosophila melanogaster (e.g., Civetta \& Ranz 2019; Hopkins, Sepil, Bonham et al. 2019; Hopkins, Sepil, Thézénas et al. 2019; Misra \& Wolfner 2020; Ravi Ram et al. 2005; Ravi Ram \& Ramesh 2003; Ravi Ram \& Wolfner 2007; Wigby et al. 2020; Wolfner 2007). Once inside the female, some of these proteins will remain bound to spermatozoa, contributing to sperm functions, and some may even interact with the already stored sperm from previous mates (e.g., Avila et al. 2011; Holman 2009; Misra \& Wolfner 2020; Ravi Ram \& Wolfner 2007; Singh et al. 2018; Wolfner 2007). Many others instead will interact intimately with female biomolecules in the reproductive tract and other organs, and are capable of changing drastically her physiology and behavior (e.g., Avila et al. 2011; Avila et al. 2016; Avila \& Wolfner 2017; Lung \& Wolfner 1999; Ravi Ram et al. 2005; Ravi Ram \& Wolfner 2007).

In Drosophila, decrease of female receptivity to mating, increase of egg production, and conformational modification of the female reproductive organs stand out among the profound changes that SFPs trigger in the female (reviewed in Avila et al. 2016). Given the conflicts of interest between males and females (and between competing males), some of the SFPs effects, while beneficial to the last-mating male, can be detrimental to the female (Chapman et al. 1995; Lung et al. 2002; Mueller et al. 2007; Wigby \& Chapman 2005). Thus, rapid antagonistic coevolution is expected between some SFPs and female-derived proteins that interact with them (e.g., Sirot et al. 2014). Nevertheless, other SFPs work synergistically with female biomolecules to facilitate fertilization or progeny production for the mutual benefit of males and females (Avila et al. 2016; Wolfner 2009). Therefore, they are expected to diverge more slowly. In fact, sequence comparisons between closely related Drosophila species revealed that some SFPs have evolved

63 extremely fast by positive selection while others are conserved by purifying selection (e.g., Almeida \& Desalle 2008; Haerty et al. 2007; Turner \& Hoekstra 2008; Wong et al. 2012). 
The biochemical classes into which SFPs typically fall (e.g., proteases, protease inhibitors, lectins, lipases, and cysteine-rich secretory proteins) seem quite conserved among Diptera, even among animals from different classes (reviewed in, e.g., Avila et al. 2016; Wigby et al. 2020). This suggests that the functional spectrum of SFPs is adaptively restricted at the molecular level. Nonetheless, a striking pattern for the vast majority of SFPs is the lack of detectable homologs among SFPs of phylogenetically distant species (Ahmed-Braimah et al. 2017; Almeida \& Desalle 2009; Davies \& Chapman 2006; Haerty et al. 2007; Mueller et al. 2005). Even though the rapid divergence of some of these proteins may hinder homology detection, the main reason behind this pattern seems to be the rapid turnover (gain and loss) of genes encoding SFPs (seminal genes) (Sirot 2019; Sirot et al. 2014). It remains unknown, however, whether a core of a particular SFPs, playing essential reproductive roles, has been conserved over long evolutionary periods. Neither do we know how new seminal genes arise so frequently, or to what extent regulatory elements of seminal genes are conserved across species.

Addressing these broad evolutionary questions requires performing multi-species comparative analyses which, in turn, requires extensive omic information on the seminal proteome of several related species. While most of the achieved findings on SFPs have been restricted to $D$. melanogaster, in recent years, the seminal proteome has been characterized in many other species, including Drosophilids. This brings up an opportunity to use the Drosophila model to address open questions on SFPs evolution and capitalize the accumulated knowledge in $D$. melanogaster.

Here, to elucidate some answers, we review the current knowledge on the evolution of Drosophila SFPs, compiled genomic data from multiple species, and performed molecular evolutionary analyses using bioinformatic tools. We structured the text into sections, each of which tackles a specific topic by presenting knowledge gaps, new insights, and future perspectives.

\section{Identification}

In D. melanogaster, as in many other dipteran species, the main secretory tissues of the male reproductive system are the accessory glands, a pair of merocrine glands attached to the anterior region of the ejaculatory duct (Avila et al. 2016; Chen 1984; Gillott 1996). While mutant males without accessory glands cannot elicit the normal postmating responses in their female mates (Kalb et al. 1993), it has long been known that ACcessory glands Proteins (ACPs) alone are sufficient for triggering these responses in virgin females (reviewed in Ravi Ram \& Wolfner 2007). In fact, the first studies on male reproductive proteins aimed to identify SFPs focusing on the male accessory glands. 
100 The very first SFP to be identified was 'Sex Peptide' (SP, also known as Acp70A). It was purified 101 from an HPLC fraction of accessory gland extracts that proved, after being injected into virgin 102 females, to reproduce the well-known postmating responses (Chen et al. 1988). The authors also 103 showed that SP gene is transcribed specifically in the male accessory glands. Afterwards, diverse 104 methods such as Expressed Sequence Tags screening, RT-PCR, subtracting hybridization, and 105 cDNA microarray hybridization allowed the identification of many other genes specifically expressed in the male accessory glands (reviewed in Chapman \& Davies 2004). Among those genes, the ones encoding proteins or peptides with a predicted signal peptide-that permits canonical merocrine secretion-were considered as candidate seminal genes (Ravi Ram \& Wolfner 2007; Swanson et al. 2001). By 2005, using this double criterion, accessory gland-specific expression and capacity to encode secretory proteins, it was possible to identify 90 putative seminal genes. Five additional seminal genes-or presumptive seminal genes-were found in other organs of the male reproductive tract: the testes, the ejaculatory duct, and the ejaculatory bulb (Cavener \& Maclntyre 1983; Dyanov \& Dzitoeva 1995; Kopantseva et al. 1990; Ludwig et al. 1991; Lung \& Wolfner 2001; Richmond et al. 1980; Saudan et al. 2002; Sheehan et al. 1979). Seven additional candidate genes were identified by mass spectrometry of tryptic peptides from accessory glands secretions (Walker et al. 2006).

Until 2008, only 22 of the predicted seminal genes were confirmed, mainly by means of immunological techniques, to be transferred to females during mating (e.g., Aigaki et al. 1991; Bertram et al. 1996; Cho et al. 1999; Coleman et al. 1995; Kopantseva et al. 1990; Lung \& Wolfner 1999, 2001; Meikle et al. 1990; Ravi Ram et al. 2005; Wong et al. 2008). In 2008, Findlay et al. conducted a proteomic screen that largely extended the list of proven SFPs. The authors used isotopic labeling of the female to distinguish, among proteins isolated from the reproductive tract of newly mated females, between female proteins and proteins transferred from unlabeled males. In this way, they confirmed 75 of the previously predicted SFPs and revealed 63 novel ones. More recently, Sepil et al. (2019) applied quantitative proteomics to identify proteins that after mating become significantly less abundant in male reproductive tissues but more abundant in the female reproductive tract, as expected precisely for SFPs. They also cross-referenced their quantification results with transcriptomic and sequence databases to obtain a list of highconfidence candidate SFPs meeting stringent multiple criteria. Some of these candidates were 130 already known as predicted or confirmed SFPs, while nine were novel discoveries (Sepil et al. 2019). While we were concluding this report, Wigby et al. (2020) combined data from these and other proteomic studies to provide a list of 292 D. melanogaster SFPs. However, the conditions they evaluated may have been too lax; according to modENCODE [implemented in FlyBase r2020_03 (Graveley et al. 2010; Thurmond et al. 2019)] and FlyAtlas2 (Leader et al. 2018), some 
tissues but in the female (e.g., FBgn0262536, FBgn0262484, and FBgn0261989), and thus, it is not clear that all these genes encode SFPs.

According to our bibliographic search, the current number of confirmed-or high-confidence candidate-non-sperm SFPs in D. melanogaster [hereafter Known Seminal Proteins (KSPs)] is 173 (see source studies in supplementary table S1). Our list includes 1) genes encoding proteins previously confirmed to be transferred by males to females during mating, 2) genes meeting the stringent multiple criteria adopted by Sepil et al. (2019), or 3) those genes more expressed in male reproductive tissues than in any other tissue (according to modENCODE and FlyAtlas2) also encoding secretory proteins found in the mating plug [according to Avila et al. (2015) and Wigby et al. (2020)]. Nonetheless, due to current methodological limitations, some other SFPs probably remain to be discovered. Given the leading role of accessory glands as suppliers of SFPs through merocrine secretion, genes that 1 ) are strongly expressed in the accessory glands and 2 ) encode secretory proteins can be considered seminal genes. Based on this expression/secretion (double) criterion, a suitable way of finding new candidate seminal genes may be to search in transcriptomic databases for genes expressed in the male accessory glands and to assess which of those genes encode secretory proteins using in silico prediction approaches.

Before searching for new candidate seminal genes, we explored to what extent $D$. melanogaster Known Seminal Genes (KSGs) meet the expression/secretion criterion by evaluating two conditions. First, we used the RNA-seq databases modENCODE (implemented in FlyBase r2020_03) and FlyAtlas2 to check which seminal genes are strongly expressed in the accessory glands. Second, we used SignalP-5.0-a deep neural network-based tool that identifies signal peptides and their cleavage sites (Almagro Armenteros et al. 2019; Nielsen et al. 1997)-to evaluate which SFPs have signal peptide required for secretion. Among the 173 KSGs, 159 (93.0\%) showed relatively high expression in the accessory glands [> 25 Reads/Fragment Per Kilobase of transcript per Million mapped reads (R/FPKM), which is within the 60-70th percentile] according to one or both databases; 156 (90.2\%) encoded a protein with a predicted signal peptide; 151 (87.2\%) meet both conditions (supplementary table S1), and; 165 (95.3\%) meet at least one of them. Most of the few genes not meeting any of these conditions are expressed specifically in the testes. These numbers not only confirm that the vast majority of SFPs are expressed in the accessory glands but also show that their secretion is mainly merocrine (but see Corrigan et al. 2014; Leiblich et al. 2012).

However, the two conditions we evaluated in the KSPs may be too lax for finding new candidate genes. For instance, accessory glands expression level could be inflated in modENCODE or FlyAtlas2, or SignalP could wrongly predict the presence of a signal peptide. Moreover, a signal peptide would only guarantee translocation into the endoplasmic reticulum followed by signal sequence cleavage. Thus, even if a gene truly meets both conditions, the protein may be retained, 
172 for instance, in the endoplasmic reticulum or the Golgi apparatus of accessory glands cells. For 173 these reasons, we decided to evaluate $D$. melanogaster genes for a more restrictive set of six 174 conditions that also relies on the expression/secretion criterion:

175 1) At least 'Very High' expression in the accessory glands (> 100 RPKM, which is within the 90th 176 percentile) according to modENCODE.

177 2) At least 'Moderately High' expression (> 25 RPKM) and expression enrichment in the accessory 178 glands (relative to other adult tissues) according to modENCODE.

179 3) At least 'Very High' expression in the accessory glands (> $100 \mathrm{FPKM}$, which is within the 90th 180 percentile) according to FlyAtlas2.

181 4) At least 'Moderately High' expression (> 25 FPKM) and expression enrichment in the accessory 182 glands (relative to whole adult male flies) according to FlyAtlas2.

5) Ability to encode a protein with a signal peptide according to SignalP.

184 6) Ability to encode a secretory protein according to DeepLoc, a prediction algorithm that uses 185 deep neural networks to predict protein localization relying on sequence information (Almagro 186 Armenteros et al. 2017). Unlike SignalP, this software differentiates between 10 subcellular 187 localizations and distinguishes proteins of the extracellular space from proteins of the secretory 188 pathway that are retained in the cell.

189 Genes fulfilling conditions 1 (or 2) and 3 (or 4) are highly (or differentially) expressed in the 190 accessory glands according to different databases, while genes fulfilling conditions 5 and 6 are 191 predicted to encode secretory proteins by different software programs. Therefore, we 192 recognized 219 D. melanogaster genes that met conditions 1 (or 2), 3 (or 4), 5, and 6 as seminal 193 gene candidates. These 219 genes included 122 KSGs, 43 previously predicted but unconfirmed 194 seminal genes, and 54 newly identified candidates (fig. 1, supplementary table S1). From the 97 195 candidates that are not among the KSGs, 46 (22 previously predicted seminal genes and 24 novel 196 discoveries) met all six conditions and were dubbed Unconfirmed High Confident Candidates 197 (UHCCs) (fig. 1, table 1).

As previously noticed, D. melanogaster seminal genes share other quite singular features: a 199 significantly biased location on autosomes, particularly on the second chromosome (Findlay et 200 al. 2008; Ravi Ram \& Wolfner 2007), and, on average, high Ka/Ks ratios (Ahmed-Braimah et al. 201 2017; Almeida \& Desalle 2008; Haerty et al. 2007; Holloway \& Begun 2004). The UHCCs resemble 202 KSGs regarding chromosomal location (fig. 2) and $K a / K s$ ratio (fig. 3). In addition, using the 203 functional annotation tool DAVID (Huang et al. 2009), we performed gene-enrichment analyses 
204 for molecular function of both UHCCs and KSGs. These analyses also revealed similarities 205 between these groups of genes: eight out of the nine (89\%) Gene Ontology (GO) terms annotated to UHCCs are among the terms annotated to KSGs, and the two most represented GO terms in the UHCCs are among the over-represented terms in the KSGs (table 2). Thus, we will henceforth refer to the 173 KSGs and the 46 UHCCs together (a total of 219 genes) as an updated list of $D$. melanogaster seminal genes.

210 Aside from D. melanogaster, the only Drosophila species in which seminal genes were extensively 211 identified are D. mojavensis (Almeida \& Desalle 2009; Kelleher et al. 2009; Wagstaff \& Begun 212 2005), D. pseudoobscura (Karr et al. 2019), D. simulans (Begun \& Lindfors 2005; Findlay et al. 213 2008; Swanson et al. 2001), D. virilis (Ahmed-Braimah et al. 2017), and D. yakuba (Begun et al. 214 2006; Findlay et al. 2008). Some (or a few) putative seminal genes were also identified in $D$. 215 biarmipes (Imamura et al. 1998), D. erecta (Begun et al. 2006), D. funebris (Baumann et al. 1975; 216 Schmidt et al. 1989), D. mayaguana (Almeida \& Desalle 2009), and D. suzukii (Ohashi et al. 1991; 217 Schmidt et al. 1993). Given the good recall of the stringent criteria we used here to identify 218 candidates, we think that other Drosophila species could be assayed for seminal genes using 219 similar criteria. Thus, further research on transcriptomic data generated from accessory glands would provide enough starting information to identify at low cost seminal genes in many species.

221 However, identifying SFPs in multiple species is only part of the equation. The evolution of the 222 seminal proteome may also diverge through changes in the expression level of seminal genes. 223 Begun and Lindfors (2005) found that transcript abundance of the seminal gene Acp24A4 224 (FBgn0051779) differs drastically between D. melanogaster and its sibling D. simulans. Findlay et 225 al. (2009) reported differences between D. melanogaster, D. simulans, and D. yakuba in the expression level and sex-specificity of several seminal genes. Similarly, Ahmed-Braimah et al. (2017) uncovered large differences in seminal transcripts abundance between members of the 228 virilis subgroup. Although these studies documented divergence between closely related species for seminal genes at the regulatory level, neither the cis nor the trans regulatory elements have been studied in depth.

Transcription is a key control point of gene expression, thus the evolution of transcription factors (TFs) that are expressed in the male accessory glands may explain much of the changes in expression of seminal genes across species. However, most of the accessory glands TFs have yet to be identified. To our knowledge, the only known accessory glands' TFs are the hox gene $A b d$ $B$ (FBgn0000015), the homeodomain transcription repressor $d v e$ (FBgn0020307), and the pairedrule gene prd (FBgn0003145), which are required for the normal development of accessory glands and the production of functional ACPs (Gligorov et al. 2013; Minami et al. 2012; Xue \& Noll 2002). Nevertheless, these genes encode pleiotropic master regulators involved in the morphogenesis of several organs and may be subjected to strong evolutionary constraints. 
240 Therefore, future research focused on the identification of accessory glands TFs will advance our 241 understanding of how seminal genes' expression has evolved.

242 It can be argued that TFs implicated in the regulation of seminal genes' expression (seminal TFs) 243 correlate with seminal genes in transcript abundance. Ayroles et al. (2011) found 224 D. 244 melanogaster genes that, besides being expressed in male reproductive tissues, showed correlated expression patterns to at least seven KSGs. Therefore, we updated this list to the current release (FlyBase r2020_03) and searched it for accessory glands TFs using an online prediction tool implemented in AnimalTFDB3.0, a comprehensive database of animal TFs (Hu et al. 2019). This first search led to the identification of eight putative seminal TFs, including the known prd and genes with unknown function (e.g., FBgn0034870, FBgn0030933, and FBgn0028480). We confirmed that all these candidates are distinctly expressed in the male accessory glands according to both modENCODE (implemented in FlyBase r2020_03) and FlyAtlas2.

Expression pattern does not necessarily correlate between seminal genes and seminal TFs. Thus, we made a second search of TFs in a more extensive list of genes including all those whose expression is enriched in the male accessory glands according to modENCODE (no less than 'Moderately High' in accessory glands but no more than 'Moderate' in any non-reproductive adult tissues) and FlyAtlas2 (accessory glands enrichment higher than 1). This second search retrieved most of the genes found in the first search plus six new candidates that have not been implicated in reproduction (table 3 ).

Next, we explored whether the candidate TFs we identified in D. melanogaster are also expressed in the male accessory glands of $D$. virilis, where accessory glands-biased transcripts were recently identified by RNA-seq (Ahmed-Braimah et al. 2017). Seven of the 14 D. melanogaster candidates showed clear homology to $D$. virilis genes with accessory glands-biased transcripts that were also predicted to encode TFs (table 3). This contrasts with the low proportion (16.9\%) of $D$. melanogaster seminal genes having homologs among $D$. virilis seminal genes. In addition, $K a / K s$ ratios estimated for the candidate seminal TFs (0.10 on average, range: $0.03-0.28$,) were lower than those estimated for seminal genes ( 0.27 on average, range: $0.02-1.51$ ) (fig. 3). These results suggest that the high turnover rate and the rapidly adaptive evolution of SFPs do not have a strong correlate in the evolution of seminal TFs.

The evolution of seminal genes' regulatory networks may follow the evolution of cis elements rather than that of TFs. However, enhancers, insulators, and promoters that are active in the male accessory glands have not been thoroughly investigated. Thus, the study of seminal TFs and their binding sites is an important area for future research. 
274 Besides TFs and their binding sites, post-transcriptional factors such as microRNAs (miRNAs) are

275 also involved in the regulation of seminal genes' expression. Recently, Mohorianu et al. (2018)

276 made an important contribution to the understanding of seminal regulatory networks by

277 assessing the role of miRNAs in the modulation of ejaculate composition. The authors found

278 evidence for the presence of several regulatory miRNAs that bind to a given sequence of the $3^{\prime}$

279 untranslated region (UTR) of seminal transcripts, likely repressing translation. Each miRNA

280 targets a specific group of seminal genes that share the corresponding 3' UTR target site, which

281 provides males with a mechanism to adjust ejaculate composition (Mohorianu et al. 2018). These

282 findings indicate that seminal genes UTRs and accessory glands miRNAs may have been involved

283 in the evolution of the seminal proteome.

284 Beyond the regulatory elements identified in D. melanogaster, causes underlying the divergence 285 of seminal genes at the regulatory level remain mostly unknown. Certainly, comparative 286 genomics will help to address this problem, however, we first need to identify the involved 287 elements in other species. Therefore, future research studying accessory glands transcriptome in 288 different Drosophila species will likely benefit this unexplored field.

\section{Turnover Rate}

291 One of the most striking characteristics of SFPs is their rich diversity, which seems to be causally 292 related, at least in part, to sexual conflict (Chapman 2008, 2018). In theory, postmating sexual 293 selection can escalate the evolutionary tension between the fitness interests of males and 294 females because male adaptations to sperm competition can be harmful to females (Chapman et al. 1995; Lung et al. 2002; Mueller et al. 2007). Selection will then favor both female traits that counteract detrimental male adaptations and male traits that respond to female resistance, potentially leading to coevolutionary arms races between male persistence and female resistance (Arnqvist 2004; Chapman et al. 2003). SFPs, by affecting female physiology and behavior, clearly influence fertilization success and sperm competitiveness. Therefore, sexual antagonistic coevolution between SFPs and the female counterparts likely accounts for the rapid divergence of seminal proteomes (Sirot et al. 2014).

As sperm competition and sexual conflict can lead to rapid adaptive divergence of orthologous SFPs, they may also promote divergence of the seminal protein repertoire through the gain of novel seminal genes as well as through seminal gene loss. On one hand, females will not be adapted to resist the action of novel SFPs. On the other hand, the expression of ancient SFPswhose action has been neutralized by females' counter-adaptations - will not be sustained by natural selection. According to this hypothesis, turnover of seminal genes would be adaptive for males because it would provide males with resources to "stay ahead" of female resistance 
309 (Chapman 2018; Sirot et al. 2014). Evidence supporting sexual conflict as a driver of seminal 310 protein evolution abounds and comes from diverse sources (reviewed in Chapman 2018; Hollis 311 et al. 2019; Sirot et al. 2014).

312 High turnover rate of seminal gene sets was first noted by Wagstaff and Begun (2005). Assaying 313 the just released D. pseudoobscura genome for orthologs of $D$. melanogaster ACP-coding genes, 314 the authors noticed an unexpectedly high proportion of absences, suggesting that an important 315 number of seminal genes are lineage-specific. Later that year, Begun and Lindfors explored the 316 presence/absence patterns of three $D$. simulans ACP-coding genes across closely related species 317 of the melanogaster subgroup, to which $D$. simulans belongs. They found that two of these genes 318 (Acp23D4 and Acp54A1) were absent in at least one species but had one to three copies in the 319 rest. Mueller et al. (2005), by performing comparative sequence analysis on 52 ACP-coding genes 320 of the melanogaster subgroup, found that 22 of them were not conserved in D. pseudoobscura. 321 Overall, these studies introduced the idea that the fraction of the genome encoding SFPs is, by 322 means of gene gain and loss, unusually dynamic.

323 With the release of the genomes of 12 Drosophila species (Drosophila 12 Genomes Consortium 324 2007), several comparative studies confirmed this pattern (e.g., Ahmed-Braimah et al. 2017; 325 Findlay et al. 2008, 2009; Haerty et al. 2007; Zhang et al. 2007). However, since too few dipteran 326 species were assayed for extensive identification of seminal genes, a comprehensive analysis to 327 trace the origin and loss of seminal genes in a phylogenetic context is lacking. Currently, we do 328 not know, for instance, to what extent orthologs of $D$. melanogaster seminal genes also encode 329 SFPs in other species of the genus. We do not know either how long ago these genes have 330 encoded SFPs in the D. melanogaster lineage. Identifying seminal genes/proteins in other 331 Drosophila species would allow to not only survey the evolutionary history of SFPs but also study 332 how new SFPs arise and how regulatory elements of seminal genes diverge between species. So 333 far, these questions have been barely explored.

334 Another question that arises is whether a core of SFPs playing essential reproductive roles has 335 been conserved throughout evolution. In such a case, these "essential SFPs", critical for 336 reproduction, should be present in a vast number of taxa. They could be searched by recognizing 337 the SFPs shared not only by closely related species but also by several phylogenetically distant 338 taxa; those shared only by closely related species would include both essential and non-essential 339 ones.

340 Intending to survey this hypothesis in Diptera, here we compiled a list of SFPs of the 341 melanogaster subgroup (those identified in D. melanogaster, D. simulans and/or D. yakuba) and 342 search it for homologs among SFPs identified in other dipteran taxa with well-known seminal 343 genes/proteins [see methodological procedures in Methods (Orthology of SFPs among Diptera)]. 
344 Taking into account that identification studies are hardly exhaustive, we only considered the 345 three outgroup taxa for which SFPs or seminal genes were identified in no less than two species by independent extensive searches. These taxa were the virilis-repleta radiation of the Drosophila 347 subgenus (that split from $D$. melanogaster $\sim 35 \mathrm{mya}$ ), tephritid fruit flies (that split from $D$. 348 melanogaster $\sim 120$ mya), and mosquitoes (that split from D. melanogaster $\sim 250 \mathrm{mya}$ ). We clustered all annotated proteins of 19 Drosophila species, including the melanogaster subgroup and the virilis-repleta radiation, in 23782 groups of orthologs (orthogroups), 196 of which have at least one seminal gene of the melanogaster subgroup. Among these 196 orthogroups 41 contain seminal genes of the virilis-repleta radiation, 11 have at least one homolog of tephritid seminal genes, and 25 have at least one homolog of mosquitoes' seminal genes (fig. 4). Caution should be taken when comparing these numbers because they relied on different homology criteria, some applied by different previous studies (supplementary table S2). However, considering that 11,298 out of the 13,969 (81\%) protein-coding genes have certainly clear homologs in mosquitoes (blastp bit score > 50), the number of SFPs shared by the four evaluated taxa seemed to be remarkably low: only two orthogroups had seminal genes of the four taxa. One of these orthogroups contains only one D. melanogaster seminal gene (FBgn0034753), which encodes a peptidyl-prolyl cis-trans isomerase. The other contains five $D$. melanogaster seminal paralogs that encode protease inhibitors with Kazal domains and belong to a tandem gene cluster located in the left arm of the second chromosome. Within this gene family, we found FBgn0266364, which was identified as a novel candidate in the present report, and FBgn0051704, which is reported in FlyBase r2020_03 as ortholog of SPINK2, a human gene implicated in male 365 infertility.

366

Although the number of taxa included in our analysis is low, the results indicate that most SFPs in Diptera are lineage-specific, which strongly suggests that most SFPs have a short evolutionary life (or diverges rapidly beyond detectable homology) and that not many-if any-have been critical for reproduction throughout Diptera evolution. Still, even if the seminal protein repertoires of the taxa we analyzed were fairly complete, our results would be far from being conclusive because homology detection across dipteran families can be inefficient for rapidly evolving seminal genes. In this sense, it would be more feasible to search for "essential SFPs" within specific groups of the Drosophila genus. However, the repertoire of SFPs is currently known for too few species. Thus, the search for "essential SFPs" within Drosophila must await more studies assaying SFPs in a wider spectrum of species.

Despite those observations and claims, gene birth and death rates were never estimated for SFPs. To obtain these estimates, we pruned the 196 orthogroups containing $D$. melanogaster seminal genes, leaving only the nine species for which genomic annotations were updated at least once [see Methods (Gene Birth and Death Rates)]. Then, duplications, losses, and orthogroup gains were identified in the gene trees of each orthogroup (fig. 5) and each event rate was estimated 
381 from the obtained figures. Taking into account divergence dates reported in Obbard et al. (2012), 382 the estimated duplication rate was 0.0097 duplications per gene per million years (/gene/my) 383 and the loss rate was 0.0122 losses/gene/my (0.0133 duplications/gene/my and 0.0212 384 losses/gene/my considering only the species of the melanogaster group). The species with the 385 greatest gene loss rate was $D$. sechellia (49 losses), which could be an artifact of genome 386 sequencing, assembly, and annotation. However, the number of protein-coding genes annotated 387 for this species is the highest in the melanogaster group and a similar pattern of high gene loss 388 was previously observed for olfactory genes in this species (Almeida et al. 2014; McBride 2007). 389 The authors associated this with $D$. sechellia specialization and endemism, which could also have 390 implications for the mating system and reproductive proteins. Regarding orthogroup gains in the 391 D. melanogaster lineage, the estimated rate was 0.0047 gains/gene/my and the total number of 392 identified events was 87. The acquisitions were inferred in the ancestors of the Sophophora 393 subgenus (25), the melanogaster group (22), the melanogaster subgroup (35), and the 394 melanogaster complex (D. melanogaster, D. simulans, and D. sechellia) (5). Interestingly, the 395 latter figure accounts for more than half the number of putative de novo genes identified by Zhou 396 et al. (2008) in the melanogaster complex.

397 Using 12 Drosophila genomes, Hahn et al. (2007) estimated a total event (gene duplications + 398 losses) rate of 0.0013 events/gene/my based on Tamura et al. (2004) divergence dates. Using the 399 same dates, we estimated for the D. melanogaster seminal genes an event rate of 0.0096 400 events/gene/my (0.0111 events/gene/my considering only the species of the melanogaster 401 group). This suggests that seminal genes' families, though they may not contain seminal genes of 402 non-D. melanogaster species, are approximately seven times more dynamic than the average 403 gene family in Drosophila.

\section{Mechanisms of Origin}

406 The high turnover rate in seminal genes/proteins repertoires implies a high proportion of novel 407 seminal genes/proteins restricted to young lineages or unique species. This facilitates studying 408 the evolution of novel genes in a common cellular background (i.e., accessory glands) in groups 409 of closely related species, where the molecular routes of gene origin are more likely traceable. 410 Thus, seminal genes provide an excellent opportunity to investigate how novel proteins and 411 biological functions emerge. Four mechanisms have been reported or proposed so far as 412 responsible for the origin of seminal genes in Drosophila: duplication of seminal genes, 413 duplication of non-seminal genes, gene co-option into the male reproductive tract, and de novo 414 evolution (reviewed in Sirot 2019). 
415 The first mechanism proposed was duplication of preexisting seminal genes (e.g., Almeida \& 416 Desalle 2009; Findlay et al. 2008; Holloway \& Begun 2004; Mueller et al. 2005; Wagstaff \& Begun 417 2005). When a seminal gene is entirely duplicated so that both copies, the new and the old, 418 encode the same SFP, ensuing mutations may lead to subfunctionalization or 419 neofunctionalization, giving rise to novel SFPs with similar amino acid sequences. Most of the 420 seminal genes encoding these proteins are located in clusters of nearby genes on the second 421 chromosome (fig. 2), showing that tandem duplication followed by mutation has played an 422 important role in the divergence of the seminal proteome. For instance, FBgn0043825, 423 FBgn0051872, and FBgn0265264 are three paralogs located in tandem on the left arm of the 424 second chromosome, which encode SFPs with triglyceride lipase activity (Mueller et al. 2005).

425 Duplication of genes that are not expressed in the male reproductive system and do not encode 426 SFPs may also be a source of novel seminal genes (Sirot 2019); if a duplicate ends up placed under 427 the control of regulatory elements driving its expression in the accessory glands, it may become 428 a new seminal gene. Genes encoding proteins that already have secretion signals are likely 429 sources for this mechanism. An example of this is the origin of the seminal gene FBgn0052833, 430 which resulted from a duplication-mediated co-option of a female-expressed gene whose original copy encodes a secretory protein of the sperm storage organs (Sirot et al. 2014). Another example comes from odorant binding proteins (OBPs), a highly dynamic family of olfactory genes that are usually expressed in the antennae. Four OBP genes, however, have been co-opted into the accessory glands exclusively in the lineage leading to the melanogaster group (Almeida et al. 2014). Interestingly, the rates of protein evolution of these genes were the highest among OBPs.

Although duplication may facilitate sequence or expression evolution because of initial redundancy (one copy can change, while the other maintains the original function), some Drosophila seminal genes seem to have arisen via gene co-option in the absence of a previous gene duplication event (Findlay et al. 2008). FBgn0262571, a D. melanogaster seminal gene exclusively expressed in the male accessory glands, belongs to a single-copy gene family (Sepil et al. 2019). Its orthologs, despite encoding proteins with secretion signal, are not within the repertoire of seminal genes in either $D$. mojavensis, D. pseudoobscura, or $D$. virilis (the only nonmelanogaster group species of the genus in which seminal genes were extensively identified). Therefore, despite not being duplicated, this gene was potentially co-opted into the accessory glands in the $D$. melanogaster lineage, during the evolution of the melanogaster group. et al. 2006; Findlay et al. 2008; Haerty et al. 2007). While sperm competition and sexual conflict may steadily select for innovation in the male ejaculate, "fitness valleys" limit the paths available for the evolution of preexisting proteins (Camps et al. 2007). In this sense, young de novo seminal genes may be less constrained and may have more opportunities to fill the emerging functional 
451 niches. Curiously, the first evidence consistent with de novo gene birth comes from studies aimed

452 to identify genes specifically expressed in Drosophila male accessory glands (Begun et al. 2006) 453 or testes (Begun et al. 2007; Zhao et al. 2014). Given the high proportion of insect seminal genes 454 without identified orthologs, de novo gene birth is believed to account for the origin of many 455 seminal genes (reviewed in Sirot 2019). So far, however, no Drosophila seminal genes have yet 456 been identified as de novo genes with high confidence, possibly because distinguishing de novo 457 birth from horizontal transfer or rapid protein divergence (which is common among seminal 458 proteins) is challenging (Zile et al. 2020).

459 Despite particular cases, a broad-scale analysis to determine the relative contribution of the 460 alternative mechanisms of origin has yet to be completed. In an attempt to discern which of the 461 mentioned mechanisms were responsible for the origin of young $D$. melanogaster SFPs [those 462 that have arisen during the evolution of the melanogaster species group, i.e., less than $\sim 25$ 463 million years ago (mya)], we identified gene families that included melanogaster group's seminal 464 genes. Given that homology detection power banishes with divergence, evaluating alternative 465 mechanisms of origin for older genes would be much more uncertain. Gene families were 466 obtained by clustering the proteins of reference proteomes of 19 Drosophila species [see 467 Methods (Seminal Gene Families)]. This analysis revealed that our set of 219 D. melanogaster 468 SFPs belong to 168 gene families. To determine which seminal genes have likely emerged after 469 the origin of the melanogaster group (which were dubbed young seminal genes), and to infer the 470 most likely mechanism of origin, we manually inspected the gene family tree of all these 168 471 gene families. Specifically, we explored the presence/absence of orthologs and paralogs, and 472 whether they had been classified as SFPs. We then applied the parsimony principle to determine, 473 according to the observed pattern, which mechanism was most likely responsible for the origin 474 of each young D. melanogaster SFP (fig. 6 illustrates our criteria). See Methods (Seminal Gene 475 Families) for a more detailed description of the applied criteria. In cases where $n$ mechanisms 476 were equally likely, we assigned " $1 / n$ genes" to each mechanism.

477 In this way, we estimated that 76 D. melanogaster seminal genes existed as seminal genes 478 (before the split from the lineage leading to $D$. pseudoobscura ( 25 mya). For 13 seminal genes, 479 we could not determine whether the origin was before or after that split since they exhibited 480 uncertain homology to sequences of outgroup or distant species. Among the remaining $130 D$. 481 melanogaster seminal genes (i.e., the tentatively young ones), we classified $\sim 27$ (20.6\%) as 482 duplicates of preexisting seminal genes, $\sim 7$ (5.3\%) as co-opted duplicates (duplicates of non483 seminal genes), $\sim 47(36.5 \%)$ as co-opted without duplication, and $\sim 49$ (37.6\%) as putative 484 orphans (fig. 7).

485 These results may give the impression that de novo emergence was responsible for the origin of 486 many D. melanogaster seminal genes. However, our approach did not contemplate all possible 
mechanisms of gene origin and may have confounded some. For instance, a non-orphan seminal gene showing fast evolution may have diverged beyond detectable homology and be construed as an orphan gene. Some of the proteomes we used may be incomplete due to potentially defective genomic annotations, which may also have led to the overestimation of taxonomically restricted genes. In consequence, the actual number of orphans among seminal genes of the melanogaster group is surely lower than the one we estimated. In fact, we could not ensure de novo status for any of the identified putative orphans [see applied criteria in Methods (De Novo Status Validation)]. Briefly, after examining several Drosophila annotated genomes, we failed to find taxonomically restricted seminal genes with syntenic homologous, reliably noncoding sequences in any outgroup species. This means that these gene families, which were initially identified as taxonomically restricted to the melanogaster group, may be classified as originating through rapid evolution (among other mechanisms) rather than de novo emergence. Therefore, the relative contribution of de novo emergence to the origin of Drosophila seminal genes may be more limited than previously thought. Gene co-option, on the other hand, appears to be the most frequent mechanism of origin.

To uncover the possible ancestral expression pattern of those few seminal genes that, according to our analysis, appear to have arisen via duplication-mediated co-option, we checked the expression pattern of the respective non-seminal paralogs. According to modENCODE (implemented in FlyBase r2020_03), these paralogs are expressed in the larval salivary gland, the adult female spermatheca, the pupal fat body, or the adult digestive system. Whether these tissues represent common sources for co-option into the seminal fluid will require further crossspecies exploration of co-opted seminal genes (for examples in other insects see Martinson et al. 2017; Meslin et al. 2015).

Alternative mechanisms of seminal genes' origin-such as exon/domain shuffling, gene fission/fusion, horizontal gene transfer, and reading-frame shift-should be explored in the future. Also, further identification of SFPs in more Drosophila species will allow for more accurate discrimination between alternative mechanisms, for dating gene origin more precisely, and for exploring gene origin in other groups.

\section{Conclusions}

Here, we provided an overview of the inter-specific divergence of Drosophila SFPs summarizing the current state of knowledge and emphasizing the intriguing aspects that are less understood. We focused on the conservation of SFPs across the order Diptera and the mechanisms of origin of Drosophila seminal genes. We not only inspected some of the main contributions to these 
521 topics but also compiled genomic information from multiple species and performed molecular

522 evolutionary analyses to address some broad questions that remain open.

523 Using reviewed criteria, we presented a novel set of high-confidence seminal protein candidates

524 for D. melanogaster and generated a database of Drosophila SFPs. We also provided, for the first

525 time, a list of accessory glands (putative or confirmed) TFs presumptively controlling the 526 expression of SFPs.

527 Two interesting patterns derive from our comparative genomic analyses. First, given the low 528 number of common SFPs found among the three inspected dipteran families, the hypothesis that 529 there is a core of indispensable, "essential SFPs" conserved across Diptera seems unlikely. 530 Second, gene co-option appears to be the most frequent mechanism accounting for the origin of 531 Drosophila seminal genes. As de novo evolution could not be ensured for any seminal gene, our 532 analysis failed to support the hypothesis that de novo emergence is a frequent mechanism of 533 origin for seminal genes.

534 Despite the insights we have gained, it is evident that characterizing the seminal proteome in 535 more species, especially in those outside the melanogaster group, is imperative to fill important 536 knowledge gaps. While proteomics on isotopic labeled flies and quantitative proteomics have 537 proven to be useful to carry out this task, our searches suggest that RNA-seq on accessory glands, 538 which is less challenging and cheaper, would provide valuable starting information.

\section{Methods}

Orthology of SFPs among Diptera

542 Supplementary table S2 summarizes the sources of the list of SFPs for each considered taxa (lists 543 are available upon request). To identify the orthologs of the SFPs identified in the melanogaster 544 group (ingroup), we employed the following strategy. First, we gathered the proteomes of 19 545 Drosophila species (see below) and used Orthofinder, a platform for comparative genomics 546 (Emms \& Kelly 2015, 2019), to cluster the proteins in groups of orthologs (orthogroups). Then, 547 we searched for the orthogroups that had any SFP of the melanogaster subgroup [i.e., the 219 of 548 D. melanogaster or those of D. simulans and/or D. yakuba identified by Findlay et al. (2008)]. The 549 input protein sequences were obtained from reference proteomes available in FlyBase, NCBI, or 550 specific genome projects' sites. The Drosophila species of the melanogaster group included in the 551 analysis were D. ananassae [dana_r1.06 (FlyBase r2020_03)], D. biarmipes [Dbia_2.0 (Richards et 552 al. unpublished, NCBI)], D. bipectinata [Dbip_2.0 (Richards et al. unpublished, NCBI)], D. elegans 553 [Dele_2.0 (Richards et al. unpublished, NCBI)], D. erecta [dere_r1.05 (FlyBase r2020_03)], D. 
eugracilis [Deug_2.0 (Richards et al. unpublished, NCBI)], D. ficusphila [Dfic_2.0 (Richards et al. unpublished, NCBI)], D. kikkawai [Dkik_2.0 (Richards et al. unpublished, NCBI)], D. mauritiana [dmauMS17_r1.0 (Nolte et al. 2013)], D. melanogaster [dmel_r6.34 (FlyBase r2020_03)], D. rhopaloa [Drho_2.0 (Richards et al. unpublished, NCBI)], D. sechellia [dsec_r1.3 (FlyBase r2020_03)], D. simulans [dsim_r2.02 (FlyBase r2020_03)], D. suzukii (Joanna C. Chiu 2020, personal communication), D. takahashii [Dtak_2.0 (Richards et al. unpublished, NCBI)], and D. yakuba [dyak_r1.05 (FlyBase 2017_03) re-annotated by Yang et al. (2018)]. Species belonging to other species groups (outgroups) were D. mojavensis [dmoj_r1.04 (FlyBase r2017_03) reannotated by Yang et al. (2018)], D. pseudoobscura [UCI_Dpse_MV25 (Liao et al. unpublished, NCBI)], and D. virilis [dvir_r1.06 (FlyBase 2017_03) re-annotated by Yang et al. (2018)]. These three species were chosen because they were the only ones outside the melanogaster group in which seminal genes were extensively studied. As Yang et al. (2018) did not annotate CDSs, we predicted for $D$. mojavensis, $D$. virilis, and $D$. yakuba one protein per gene with RefProt pipeline (Revale \& Hurtado, available upon request), which is based on TransDecoder (Haas et al. 2013), Blast (Altschul et al. 1990), HMMER (hmmer.org), and several inhouse R scripts (R-project.org). In our experience, Orthofinder has limited recall when clustering sequences of very distantly related species. Therefore, to recognize orthogroups with SFPs of species outside Drosophila (Aedes aegypti, Aedes albopictus, Anopheles gambiae, Bactrocera dorsalis, and Ceratitis capitata) we relied on previous orthology assignments based on Blast (supplementary table S2). We considered a SFP to be shared between melanogaster subgroup and any given outgroup if the protein was clustered together with an outgroup SFP in the same orthogroup.

\section{Molecular Evolutionary Analyses}

Estimates of the ratio between the rate of non-synonymous substitution $(K a)$ and the rate of synonymous substitutions $(K s)$ can be used as a proxy to investigate the evolutionary forces that shape the evolution of proteins. Close to zero ratios are associated with purifying selection, whereas ratios close or higher than one mean that the gene evolves under neutrality or that some codons are positively selected. We employed PAML-4.8 (Yang 2007) to obtain $\omega$, a likelihood-based estimator of $K a / K s$, for each orthogroup.

\section{Gene Birth and Death Rates}

We pruned the 196 orthogroups containing D. melanogaster SFP-coding genes (see above) to include only those species with updated genome annotations, leaving in this way the orthologs of D. melanogaster, D. simulans, D. sechellia, D. erecta, D. yakuba, D. ananassae, D. 
pseudoobscura, D. mojavensis, and D. virilis. Then we employed the program Notung-2.9.1.5

589 (Chen et al. 2000; Darby et al. 2017) to identify gene duplications, losses, and de novo gains in 590 each orthogroup by comparing gene trees with the species tree. To be conservative and avoid 591 overestimation, we edited the Notung results to remove duplications and losses when there was 592 an even number of genes per species. With the total number of each of these events for each 593 branch of the Drosophila phylogeny, we estimated per gene rates by dividing the number of 594 events by the number of genes in the ancestral branches. These events were summed across all 595 branches and the sum was divided by the total phylogeny time to obtain the rates using the 596 formulas described in Vieira et al. (2007). A gene gain was identified for each orthogroup 597 exclusive of a monophyletic clade.

600 Since Orthofinder inference relies on reciprocal best alignment hits, some paralogous sequences 601 ended up grouped in separate orthogroups. Thus, with the aim of identifying paralogous 602 orthogroups, we compared D. melanogaster sequences clustered in different orthogroups using 603 Blastp. We then merged orthogroups with aligned sequences into more inclusive gene families. 604 Since we used a conservative bit score cutoff of 80 for filtering hits, the number of recognized 605 gene families probably represent an upper bound of the actual number. Our objective was to 606 determine the origin of $D$. melanogaster seminal genes that had emerged during the evolution 607 of the melanogaster group (i.e., after the split from the lineage leading to D. pseudoobscura), so 608 we considered the species belonging to other groups as outgroups. We then used the gene trees 609 generated by Orthofinder to investigate the origins of the melanogaster group SFPs. Within each 610 orthogroup, the last common ancestor gene between an outgroup seminal gene and a $D$. 611 melanogaster seminal gene was considered as a seminal gene. Similarly, the last common 612 ancestor gene at the root of any orthogroup containing homologs to seminal genes of tephritids 613 or mosquitoes was also considered as a seminal gene. With these considerations, we inferred the 614 most likely mechanism of origin of each $D$. melanogaster seminal gene by manually inspecting 615 the respective gene family tree. Specifically, we explored the presence/absence of orthologs and 616 paralogs among species of the melanogaster group and outgroups applying the parsimony 617 principle over gene gain/loss events (fig. 6). In this way, we first distinguished between "ancient" 618 (those that had emerged before the split from the lineage leading to $D$. pseudoobscura, 25 mya) 619 and tentatively young (those lacking homologs among outgroup seminal genes, that have likely 620 emerged after the split from the lineage leading to D. pseudoobscura) D. melanogaster seminal 621 genes. Then, we classified tentatively young seminal genes into the following four categories: 622 duplicated, co-opted after being duplicated, co-opted without being duplicated, and orphan. In 623 those cases where $n$ mechanisms were equally likely, we assigned " $1 / n$ genes" to each 
624 mechanism. Some D. melanogaster proteins may have evolved very rapidly, hindering homology

625 detection. Thus, in the case of SFPs classified as orphan with our approach, we evaluated distant homology by comparing $D$. melanogaster SFPs to non-redundant proteins sequences from NCBI

627 databases using Blastp (blast.ncbi.nlm.nih.gov). In this case, we admitted hits (bit score > 39)

628 against sequences of any Diptera: those with any bit score higher than 50 were considered to 629 reflect homology while those with bit scores between 39 and 50 were considered uncertain. Also, 630 for each apparent orphan seminal gene, we checked manually the absence of syntenic open 631 reading frames encoding similar proteins (Blastp: bit score $>39$ or positives $>60 \%$ ) in the $D$. 632 pseudobscura genome by using the Ensembl Metazoa genome browser (Howe et al. 2019).

\section{De Novo Status Validation}

To validate the de novo status of the putative orphans, we used the conservative criteria applied by Zile et al. (2020). Briefly, as de novo genes should have syntenic, homologous noncoding sequences in closely related outgroup species, we inspected each orphan candidate for syntenic, homologous noncoding sequences in well-annotated genomes of outgroup species. Particularly, we examined the latest public assemblies for D. anananassae [DanaRS2.1 (Zhang et al.unpublished, $\mathrm{NCBI}$ )], D. elegans [Dele_2.0 (Richards et al. unpublished, NCBI)], D. erecta [DereRS2 (Zhang et al.unpublished, NCBI)], D. pseudoobscura [UCl_Dpse_MV25 (Liao et al. unpublished, NCBI)], D. simulans [Prin_Dsim_3.0 (Pinharanda et al. unpublished, NCBI)], D.

643 suzukii [LBDM_Dsuz_2.1.pri (Paris et al. unpublished, NCBI)], and D. yakuba 644 [Prin_Dyak_Tai18E2_2.0 (Reilly et al. unpublished, NCBI)]. For instance, for a gene family restricted to the melanogaster complex (D. melanogaster, D. sechellia and D. simulans), any species outside this complex (i.e., D. ananassae, D. elegans, D. erecta, D. pseudoobscura, D.

647 suzukii and D. yakuba) was considered an outgroup. Thus, for each gene family having orphan 648 candidates, Blastn searches were applied to search the syntenic genomic regions of the outgroup 649 genomes for homologous sequences (bit score $>39$ or identities $>60 \%$ ). The found homologous 650 syntenic sequences showing evidence of being transcribed (i.e., evidence from RNA-Seq 651 alignment data) were searched-employing Blastp searches-for the absence of homologous 652 open reading frames (bit score $<39$ and positives $<60 \%$ ).

\section{Acknowledgments}

655 We thank Nicolás Moreyra and Agustina Sztyrle for comments that helped in different stages of 656 the present study, and Joanna Chiu (University of California, Davis) for providing access to the $D$. 
657

658

659

660

661

662

663

664

665

666

667

668

669

670

671

672

673

674

675

676

677

678

679

680

681

682

683

684

685

686

suzukii 2.0 genome. This work was supported by the Agencia Nacional de Promoción Científica y Técnica through grants awarded to $\mathrm{JH}$ and $\mathrm{EH}$.

\section{Authors' Contributions}

$\mathrm{JH}$ conceived and designed the study, compiled and analyzed the data, and took the lead in writing the manuscript. FCA was involved in planning the work and analysis design; she also estimated rates of molecular evolution and gene gain/loss. SAB performed functional annotations and designed the figures. SR helped integrate genomic information and predict protein sequences. EH was involved in planning the work and supervised the project. All authors discussed the results and contributed to the final manuscript.

\section{Supplementary Material}

Table S1. List of D. melanogaster seminal genes. As KSGs we included genes encoding proteins previously confirmed to be transferred by males into females during mating, those meeting stringent multiple criteria that indicate so according to Sepil et al. (2019), or those expressed in male reproductive tissues more than in any other tissue (according to modENCODE and FlyAtlas2) also encoding secretable proteins found in the mating plug [according to Avila et al. (2015) and Wigby et al. (2020)]. As candidates, we included our novel candidates as well as previously predicted seminal genes. We excluded genes expressed specifically in the testes (according to FlyAtlas2) that encode sperm proteins (Wigby et al. 2020), those candidates proposed only by Wigby et al. (2020) that show low expression in male reproductive tissues and higher expression in other male and female tissues (according to modENCODE and FlyAtlas2), and those proposed only by Ayroles et al. (2011) that do not encode secretable proteins (signalP). The evaluated conditions for the expression/secretion criterion and sources that previously identified the gene as seminal are shown for each gene (see supplementary references).

Table S2. SFPs of the melanogaster subgroup, the virilis-repleta radiation, tephritids, and mosquitoes. Sources and methods used to compile the list are summarized for each considered species (see supplementary references).

Data Availability 
687 Despite no new data were generated in support of this research, the compiled information and 688 data underlying our analyses are available in the article, in its online supplementary material, and/or at the open-access databases duly mentioned in the text.

690

691

\section{References}

692

Ahmed-Braimah YH, Unckless RL, Clark AG. 2017. Evolutionary dynamics of male reproductive genes in the Drosophila virilis subgroup. G3 Genes, Genomes, Genet. 7:3145-3155. doi:

Aigaki T, Fleischmann I, Chen PS, Kubli E. 1991. Ectopic expression of sex peptide alters reproductive behavior of female D. melanogaster. Neuron. 7:557-563. doi: 10.1016/08966273(91)90368-A.

698

699

700

701

702

703

704

705

706

707

708

709

710

711

712

713

714

715

716

Almagro Armenteros JJ et al. 2019. SignalP 5.0 improves signal peptide predictions using deep neural networks. Nat. Biotechnol. 37:420-423. doi: 10.1038/s41587-019-0036-z.

Almagro Armenteros JJ, Sønderby CK, Sønderby SK, Nielsen H, Winther O. 2017. DeepLoc: prediction of protein subcellular localization using deep learning. Bioinformatics. 33:3387-3395. doi: 10.1093/bioinformatics/btx431.

Almeida FC, Desalle R. 2008. Evidence of adaptive evolution of accessory gland proteins in closely related species of the Drosophila repleta group. Mol. Biol. Evol. 25:2043-2053. doi: 10.1093/molbev/msn155.

Almeida FC, Desalle R. 2009. Orthology, function and evolution of accessory gland proteins in the Drosophila repleta group. Genetics. 181:235-245. doi: 10.1534/genetics.108.096263.

Almeida FC, Sánchez-Gracia A, Campos JL, Rozas J. 2014. Family size evolution in Drosophila chemosensory gene families: A comparative analysis with a critical appraisal of methods. Genome Biol. Evol. 6:1669-1682. doi: 10.1093/gbe/evu130.

Altschul SF, Gish W, Miller W, Myers EW, Lipman DJ. 1990. Basic local alignment search tool. J. Mol. Biol. 215:403-410. doi: 10.1016/S0022-2836(05)80360-2.

Arnqvist G. 2004. Sexual conflict and sexual selection: lost in the chase. Evolution. 58:1383-1393. doi: 10.1111/j.0014-3820.2004.tb01716.x.

Avila FW et al. 2015. Retention of ejaculate by Drosophila melanogaster females requires the male-derived mating plug protein PEBme. Genetics. 200:1171-1179. doi: 
Avila FW et al. 2016. Nature and functions of glands and ducts in the Drosophila reproductive tract. In: Extracellular Composite Matrices in Arthropods. Cohen, E \& Moussian, B, editors. Switzerland: Springer International Publishing pp. 411-444. doi: 10.1007/978-3-319-40740-1.

Avila FW, Sirot LK, LaFlamme BA, Rubinstein CD, Wolfner MF. 2011. Insect seminal fluid proteins: identification and function. Annu. Rev. Entomol. 56:21-40. doi: 10.1146/annurev-ento-120709144823.

Avila FW, Wolfner MF. 2017. Cleavage of the Drosophila seminal protein Acp36DE in mated females enhances its sperm storage activity. J Insect Physiol. 101:66-72. doi:10.1016/j.jinsphys.2017.06.015.

Ayroles JF, Laflamme BA, Stone EA, Wolfner MF, Mackay TFC. 2011. Functional genome annotation of Drosophila seminal fluid proteins using transcriptional genetic networks. Genet. Res. 93:387-395. doi: 10.1017/S0016672311000346.

Baumann H, Wilson KJ, Chen PS, Humbel RE. 1975. The amino-acid sequence of a peptide (PS-1) from Drosophila funebris: a paragonial peptide from males which reduces the receptivity of the female. Eur. J. Biochem. 52:521-529. doi: 10.1111/j.1432-1033.1975.tb04023.x. subgroup of Drosophila. Mol. Biol. Evol. 22:2010-2021. doi: 10.1093/molbev/msi201.

Begun DJ, Lindfors HA, Kern AD, Jones CD. 2007. Evidence for de novo evolution of testisexpressed genes in the Drosophila yakuba/Drosophila erecta clade. Genetics. 176:1131-1137. doi: 10.1534/genetics.106.069245.

Begun DJ, Lindfors HA, Thompson ME, Holloway AK. 2006. Recently evolved genes identified from Drosophila yakuba and D. erecta accessory gland expressed sequence tags. Genetics. 172:16751681. doi: 10.1534/genetics.105.050336.

Bertram MJ, Neubaum DM, Wolfner MF. 1996. Localization of the Drosophila male accessory 742 gland protein Acp36DE in the mated female suggests a role in sperm storage. Insect Biochem. 743 Mol. Biol. 26:971-980. doi: 10.1016/s0965-1748(96)00064-1.

744 Camps M, Herman A, Loh E, Loeb LA. 2007. Genetic constraints on protein evolution. Crit. Rev. 745 Biochem. Mol. Biol. 42:313-326. doi: 10.1080/10409230701597642. 
747 Drosophila melanogaster. Proc. Natl. Acad. Sci. U. S. A. 80:6286-6288. doi: $748 \quad 10.1073 /$ pnas.80.20.6286.

749 Chapman T. 2018. Sexual conflict: Mechanisms and emerging themes in resistance biology. Am. 750 Nat. 192:217-229. doi: 10.1086/698169.

751 Chapman $T$ et al. 2003. The sex peptide of Drosophila melanogaster: Female post-mating 752 responses analyzed by using RNA interference. Proc. Natl. Acad. Sci. U. S. A. 100:9923-9928. doi: 753 10.1073/pnas.1631635100.

754 Chapman T. 2008. The soup in my fly: Evolution, form and function of seminal fluid proteins. PLoS 755 Biol. 6:1379-1382. doi: 10.1371/journal.pbio.0060179.

756 Chapman T, Davies SJ. 2004. Functions and analysis of the seminal fluid proteins of male 757 Drosophila melanogaster fruit flies. Peptides. 25:1477-1490. doi: 758 10.1016/j.peptides.2003.10.023.

759 Chapman T, Liddle LF, Kalb JM, Wolfner MF, Partridge L. 1995. Cost of mating in Drosophila 760 melanogaster females is mediated by male accessory gland products. Nature. 373:241-244. doi: $761 \quad 10.1038 / 373241 \mathrm{a} 0$.

762 Chen K, Durand D, Farach-Colton M. 2000. NOTUNG: a program for dating gene duplications and 763 optimizing gene family trees. J. Comput. Biol. 7:429-447. doi: 10.1089/106652700750050871.

764 Chen PS et al. 1988. A male accessory gland peptide that regulates reproductive behavior of 765 female D. melanogaster. Cell. 54:291-298. doi: 10.1016/0092-8674(88)90192-4.

766 Chen S. 1984. Biochemistry of Insect Male Accessory Glands. Annu. Rev. Entomol. 29:233-255.

767 Cho KS et al. 1999. A 45-kDa cAMP-dependent phosphoprotein which is related to the product of 768 Mst57Dc in Drosophila melanogaster. Insect Biochem. Mol. Biol. 29:701-710. doi: 769 10.1016/S0965-1748(99)00046-6.

770 Civetta A, Ranz JM. 2019. Genetic factors influencing sperm competition. Front. Genet. 10:820. 771 doi: 10.3389/fgene.2019.00820.

772 Coleman S, Drähn B, Petersen G, Stolorov J, Kraus K. 1995. A Drosophila male accessory gland 773 protein that is a member of the serpin superfamily of proteinase inhibitors is transferred to 774 females during mating. Insect Biochem. Mol. Biol. 25:203-207. doi: 10.1016/0965775 1748(94)00055-M.

776 Corrigan L et al. 2014. BMP-regulated exosomes from Drosophila male reproductive glands 
reprogram female behavior. J. Cell Biol. 206:671-688. doi: 10.1083/jcb.201401072.

778

779

780

781

782

783

784

785

786

787

788

789

790

791

792

793

794

795

796

797

798

799

800

801

802

803

804

805

806

Darby CA, Stolzer M, Ropp PJ, Barker D, Durand D. 2017. Xenolog classification. Bioinformatics. 33:640-649. doi: 10.1093/bioinformatics/btw686.

Davies SJ, Chapman T. 2006. Identification of genes expressed in the accessory glands of male Mediterranean Fruit Flies (Ceratitis capitata). Insect Biochem. Mol. Biol. 36:846-856. doi: 10.1016/j.ibmb.2006.08.009.

Drosophila 12 Genomes Consortium. 2007. Evolution of genes and genomes on the Drosophila phylogeny. Nature. 450:203-218. doi: 10.1038/nature06341.

Dyanov HM, Dzitoeva SG. 1995. Method for attachment of microscopic preparations on glass for in situ hybridization, PRINS and in situ PCR studies. Biotechniques. 18:822-826.

Emms DM, Kelly S. 2019. OrthoFinder: Phylogenetic orthology inference for comparative genomics. Genome Biol. 20:1-14. doi: 10.1186/s13059-019-1832-y.

Emms DM, Kelly S. 2015. OrthoFinder: solving fundamental biases in whole genome comparisons dramatically improves orthogroup inference accuracy. Genome Biol. 16:1-14. doi: 10.1186/s13059-015-0721-2.

Findlay GD, MacCoss MJ, Swanson WJ. 2009. Proteomic discovery of previously unannotated, rapidly evolving seminal fluid genes in Drosophila. Genome Res. 19:886-895. doi: 10.1101/gr.089391.108.

Findlay GD, Yi X, Maccoss MJ, Swanson WJ. 2008. Proteomics reveals novel Drosophila seminal fluid proteins transferred at mating. PLoS Biol. 6:e178. doi: 10.1371/journal.pbio.0060178.

Gillott C. 1996. Male insect accessory glands: Functions and control of secretory activity. Invertebr. Reprod. Dev. 30:199-205. doi: 10.1080/07924259.1996.9672546.

Gligorov D, Sitnik JL, Maeda RK, Wolfner MF, Karch F. 2013. A novel function for the hox gene $A b d-b$ in the male accessory gland regulates the long-term female post-mating response in Drosophila. PLoS Genet. 9(3):e1003395. doi: 10.1371/journal.pgen.1003395.

Graveley BR et al. 2010. The D. melanogaster transcriptome: modENCODE RNA-Seq data. http://www.modencode.org/celniker/.

Haas BJ et al. 2013. De novo transcript sequence reconstruction from RNA-seq using the Trinity platform for reference generation and analysis. Nat. Protoc. 8:1494-1512. doi: 10.1038/nprot.2013.084. 
807 Haerty W et al. 2007. Evolution in the fast lane: Rapidly evolving sex-related genes in Drosophila. 808 Genetics. 177:1321-1335. doi: 10.1534/genetics.107.078865.

809 Hahn MW, Han MV, Han S-G. 2007. Gene family evolution across 12 Drosophila genomes. PLoS 810 Genet. 3:e197. doi: 10.1371/journal.pgen.0030197.

811 Hollis B et al. 2019. Sexual conflict drives male manipulation of female postmating responses in 812 Drosophila melanogaster. Proc. Natl. Acad. Sci. U. S. A. 116:8437-8444. doi: 813 10.1073/pnas.1821386116.

814 Holloway AK, Begun DJ. 2004. Molecular evolution and population genetics of duplicated accessory gland protein genes in Drosophila. Mol. Biol. Evol. 21:1625-1628. doi: $10.1093 / \mathrm{molbev} / \mathrm{msh} 195$.

Holman L. 2009. Drosophila melanogaster seminal fluid can protect the sperm of other males. Funct. Ecol. 23:180-186. doi: 10.1111/j.1365-2435.2008.01509.x.

819 Hopkins BR, Sepil I, Bonham S et al. 2019. BMP signaling inhibition in Drosophila secondary cells 820 remodels the seminal proteome and self and rival ejaculate functions. Proc. Natl. Acad. Sci. U. S. 821 A. 116(49):24719-24728. doi: 10.1073/pnas.1914491116.

822 Hopkins BR, Sepil I, Thézénas ML et al. 2019. Divergent allocation of sperm and the seminal 823 proteome along a competition gradient in Drosophila melanogaster. Proc. Natl. Acad. Sci. U. S. A. 824 116(36):17925-17933. doi: 10.1073/pnas.1906149116.

825 Howe KL et al. 2019. Ensembl Genomes 2020-enabling non-vertebrate genomic research. 826 Nucleic Acids Res. 48:D689-D695. doi: 10.1093/nar/gkz890.

$827 \mathrm{Hu} \mathrm{H}$ et al. 2019. AnimalTFDB 3.0: a comprehensive resource for annotation and prediction of 828 animal transcription factors. Nucleic Acids Res. 47:D33-D38. doi: 10.1093/nar/gky822.

829 Huang DW, Sherman BT, Lempicki RA. 2009. Systematic and integrative analysis of large gene lists using DAVID bioinformatics resources. Nat. Protoc. 4:44-57. doi: 10.1038/nprot.2008.211.

831 Imamura M, Haino-Fukushima K, Aigaki T, Fuyama Y. 1998. Ovulation stimulating substances in 832 Drosophila biarmipes males: Their origin, genetic variation in the response of females, and 833 molecular characterization. Insect Biochem. Mol. Biol. 28:365-372. doi: 10.1016/S0965834 1748(98)00004-6.

835 Kalb JM, Dibenedetto AJ, Wolfnert MF. 1993. Probing the function of Drosophila melanogaster 836 accessory glands by directed cell ablation. Proc. Natl. Acad. Sci. U. S. A. 90:8093-8097. 
837 Karr TL, Southern H, Rosenow MA, Gossmann TI, Snook RR. 2019. The old and the new: Discovery 838 proteomics identifies putative novel seminal fluid proteins in Drosophila. Mol. Cell. Proteomics. 839 18:S23-S33. doi: 10.1074/mcp.RA118.001098.

840 Kelleher ES, Watts TD, LaFlamme BA, Haynes PA, Markow TA. 2009. Proteomic analysis of 841 Drosophila mojavensis male accessory glands suggests novel classes of seminal fluid proteins. 842 Insect Biochem. Mol. Biol. 39:366-371. doi: 10.1016/j.ibmb.2009.03.003.

843 Kopantseva MR et al. 1990. The proteins of the ejaculatory bulbs in different species of 844 Drosophila. Zh. Obshch. Biol. 51:125-140.

845 Leader DP, Krause SA, Pandit A, Davies SA, Dow JAT. 2018. FlyAtlas 2: a new version of the 846 Drosophila melanogaster expression atlas with RNA-Seq, miRNA-Seq and sex-specific data. 847 Nucleic Acids Res. 46:D809-D815. doi: 10.1093/nar/gkx976.

848 Lefevre G. 1976. A photographic representation and interpretation of the polytene chromosomes 849 of Drosophila melanogaster salivary glands. In: The Genetics and Biology of Drosophila. 850 Ashburner, M \& Novitski, E, editors. Vol. la London: Academic Press pp. 31-66.

851 Leiblich A et al. 2012. Bone morphogenetic protein- and mating-dependent secretory cell growth 852 and migration in the Drosophila accessory gland. Proc. Natl. Acad. Sci. U. S. A. 109:19292-19297. 853 doi: 10.1073/pnas.1214517109.

854 Ludwig MZ et al. 1991. Genetic control and expression of the major ejaculatory bulb protein PEB855 me in Drosophila melanogaster. Biochem. Genet. 29:215-240.

856 Lung $\mathrm{O}$ et al. 2002. The Drosophila melanogaster seminal fluid protein Acp62F is a protease 857 inhibitor that is toxic upon ectopic expression. Genetics. 160:211-224.

858 Lung O, Wolfner MF. 1999. Drosophila seminal fluid proteins enter the circulatory system of the 859 mated female fly by crossing the posterior vaginal wall. Insect Biochem. Mol. Biol. 29:1043-1052. 860 doi: 10.1016/S0965-1748(99)00078-8.

861 Lung O, Wolfner MF. 2001. Identification and characterization of the major Drosophila 862 melanogaster mating plug protein. Insect Biochem. Mol. Biol. 31:543-551. doi: 10.1016/S0965863 1748(00)00154-5.

864 Martinson EO, Mrinalini, Kelkar YD, Chang C, Werren JH. 2017. The evolution of venom by co865 option of single copy genes. Curr. Biol. 27(13):2007-2013.e8. doi:10.1016/j.cub.2017.05.032. 
867 in Drosophila sechellia. Proc. Natl. Acad. Sci. U. S. A. 104:4996-5001. doi: 868 10.1073/pnas.0608424104.

869 Meikle DB, Sheehan KB, Phillis DM, Richmond RC. 1990. Localization and longevity of seminal870 fluid esterase 6 in mated female Drosophila melanogaster. J. Insect Physiol. 36:93-101. doi: 871 10.1016/0022-1910(90)90179-J.

872 Meslin C et al. 2015. Digestive organ in the female reproductive tract borrows genes from 873 multiple organ systems to adopt critical functions. Mol. Biol. Evol. 32(6):1567-1580. doi: 874 10.1093/molbev/msv048.

875 Minami R et al. 2012. The homeodomain protein defective proventriculus is essential for male 876 accessory gland development to enhance fecundity in Drosophila. PLoS One. 7:e32302. doi: 877 10.1371/journal.pone.0032302.

878 Misra S, Wolfner MF. 2020. Drosophila seminal sex peptide associates with rival as well as own 879 sperm, providing SP function in polyandrous females. Elife. 9:e58322. doi: 10.7554/eLife.58322.

880 Mohorianu I, Fowler EK, Dalmay T, Chapman T. 2018. Control of seminal fluid protein expression 881 via regulatory hubs in Drosophila melanogaster. Proc. R. Soc. B Biol. Sci. 285:20181681. doi: $88210.1098 / \mathrm{rspb} .2018 .1681$.

883 Mueller JL et al. 2005. Cross-species comparison of Drosophila male accessory gland protein 884 genes. Genetics. 171:131-143. doi: 10.1534/genetics.105.043844.

885 Mueller JL, Page JL, Wolfner MF. 2007. An ectopic expression screen reveals the protective and 886 toxic effects of Drosophila seminal fluid proteins. Genetics. 175:777-783. doi: 887 10.1534/genetics.106.065318.

888 Nielsen H, Engelbrecht J, Brunak S, von Heijne G. 1997. Identification of prokaryotic and 889 eukaryotic signal peptides and prediction of their cleavage sites. Protein Eng. Des. Sel. 10:1-6. 890 doi: 10.1093/protein/10.1.1.

891 Nolte V, Pandey RV, Kofler R, Schlötterer C. 2013. Genome-wide patterns of natural variation 892 reveal strong selective sweeps and ongoing genomic conflict in Drosophila mauritiana. Genome 893 Res. 23:99-110. doi: 10.1101/gr.139873.112.

894 Obbard DJ et al. 2012. Estimating divergence dates and substitution rates in the Drosophila 895 phylogeny. Mol. Biol. Evol. 29:3459-3473. doi: 10.1093/molbev/mss150.

896 Ohashi YY, Haino-Fukushima K, Fuyama Y. 1991. Purification and characterization of an ovulation 
897

898

899

900

901

902

903

904

905

906

907

908

909

910

911

912

913

914

915

916

917

918

919

920

921

922

923

924

925

926

stimulating substance from the male accessory glands of Drosophila suzukii. Insect Biochem. 21:413-419. doi: 10.1016/0020-1790(91)90008-3.

Ramm SA. 2020. Seminal fluid and accessory male investment in sperm competition. Phil. Trans. R. Soc. B. 375: 20200068. doi: 10.1098/rstb.2020.0068.

Ravi Ram K, Ji S, Wolfner MF. 2005. Fates and targets of male accessory gland proteins in mated female Drosophila melanogaster. Insect Biochem. Mol. Biol. 35:1059-1071. doi: 10.1016/j.ibmb.2005.05.001.

Ravi Ram K, Ramesh SR. 2003. Male accessory gland proteins in Drosophila: A multifacet field. Indian J. Exp. Biol. 41:1372-1383.

Ravi Ram K, Wolfner MF. 2007. Seminal influences: Drosophila Acps and the molecular interplay between males and females during reproduction. Integr. Comp. Biol. 47:427-445. doi: 10.1093/icb/icm046.

Richmond RC, Gilbert DG, Sheehan KB, Gromko MH, Butterworth FM. 1980. Esterase 6 and reproduction in Drosophila melanogaster. Science. 207:1483-1485. doi: 10.1126/science.6767273.

Saudan P et al. 2002. Ductus ejaculatorius peptide 99B (DUP99B), a novel Drosophila melanogaster sex-peptide pheromone. Eur. J. Biochem. 269:989-997. doi: 10.1046/j.00142956.2001.02733.x.

Schmidt T et al. 1993. Drosophila suzukii contains a peptide homologous to the Drosophila melanogaster sex-peptide and functional in both species. Insect Biochem. Mol. Biol. 23:571-579. doi: 10.1016/0965-1748(93)90030-V.

Schmidt T, Stumm-Zollinger E, Chen PS, Böhlen P, Stone SR. 1989. A male accessory gland peptide with protease inhibitory activity in Drosophila funebris. J. Biol. Chem. 264:9745-9749.

Sepil I et al. 2019. Quantitative proteomics identification of seminal fluid proteins in male Drosophila melanogaster. Mol. Cell. Proteomics. 18:S46-S58. doi: 10.1074/mcp.RA118.000831.

Sheehan K, Richmond RC, Cochrane BJ. 1979. Studies of esterase 6 in Drosophila melanogaster. III. The developmental pattern and tissue distribution. Insect Biochem. 9:443-450. doi: 10.1016/0020-1790(79)90062-3.

Singh A et al. 2018. Long-term interaction between Drosophila sperm and sex peptide is mediated by other seminal proteins that bind only transiently to sperm. Insect Biochem. Mol. Biol. 102:43- 
51. doi: 10.1016/j.ibmb.2018.09.004.

928 Sirot LK. 2019. On the evolutionary origins of insect seminal fluid proteins. Gen. Comp. 929 Endocrinol. 278:104-111. doi: 10.1016/j.ygcen.2019.01.011.

930 Sirot LK, Wong A, Chapman T, Wolfner MF. 2014. Sexual conflict and seminal fluid proteins: A 931 dynamic landscape of sexual interactions. Cold Spring Harb. Perspect. Biol. 7:a017533. doi: 932 10.1101/cshperspect.a017533.

933 Swanson WJ, Clark AG, Waldrip-Dail HM, Wolfner MF, Aquadro CF. 2001. Evolutionary EST 934 analysis identifies rapidly evolving male reproductive proteins in Drosophila. Proc. Natl. Acad. Sci. U. S. A. 98:7375-7379. doi: 10.1073/pnas.131568198.

936 Tamura K, Subramanian S, Kumar S. 2004. Temporal patterns of fruit fly (Drosophila) evolution 937 revealed by mutation clocks. Mol. Biol. Evol. 21:36-44. doi: 10.1093/molbev/msg236.

938 Thurmond J et al. 2019. FlyBase 2.0: the next generation. Nucleic Acids Res. 47:D759-D765. doi: 939 10.1093/nar/gky1003.

940 Turner LM, Hoekstra HE. 2008. Causes and consequences of the evolution of reproductive 941 proteins. Int. J. Dev. Biol. 52:769-780. doi: 10.1387/ijdb.082577lt.

942 Vieira FG, Sánchez-Gracia A, Rozas J. 2007. Comparative genomic analysis of the odorant-binding 943 protein family in 12 Drosophila genomes: purifying selection and birth-and-death evolution. 944 Genome Biol. 8:R235. doi: 10.1186/gb-2007-8-11-r235.

945 Wagstaff BJ, Begun DJ. 2005. Comparative genomics of accessory gland protein genes in 946 Drosophila melanogaster and D. pseudoobscura. Mol. Biol. Evol. 22:818-832. doi: 947 10.1093/molbev/msi067.

948 Walker MJ et al. 2006. Proteomic identification of Drosophila melanogaster male accessory gland 949 proteins, including a pro-cathepsin and a soluble $\gamma$-glutamyl transpeptidase. Proteome Sci. 4:1950 10. doi: 10.1186/1477-5956-4-9.

951 Wigby S et al. 2020. The Drosophila seminal proteome and its role in postcopulatory sexual 952 selection. Trans R Soc Lond B Biol Sci. 375(1813):20200072. doi: 10.1098/rstb.2020.0072.

953 Wigby S, Chapman T. 2005. Sex peptide causes mating costs in female Drosophila melanogaster. 954 Curr. Biol. 15(4):316-21. doi: 10.1016/j.cub.2005.01.051.

955 Wolfner MF. 2009. Battle and ballet: Molecular interactions between the sexes in Drosophila. J. 956 Hered. 100:399-410. doi: 10.1093/jhered/esp013. 
957 Wolfner MF. 2007. 'S.P.E.R.M.' (seminal proteins (are) essential reproductive modulators): the 958 view from Drosophila. Soc. Reprod. Fertil. Suppl. 65:183-199.

959 Wong A et al. 2008. A role for Acp29AB, a predicted seminal fluid lectin, in female sperm storage 960 in Drosophila melanogaster. Genetics. 180:921-931. doi: 10.1534/genetics.108.092106.

961 Wong A, Turchin MC, Wolfner MF, Aquadro CF. 2012. Temporally variable selection on 962 proteolysis-related reproductive tract proteins in Drosophila. Mol. Biol. Evol. 29:229-238. doi: 963 10.1093/molbev/msr197.

964 Xue L, Noll M. 2002. Dual role of the Pax gene paired in accessory gland development of 965 Drosophila. Development. 129:339-346.

966 Yang $\mathrm{H}$ et al. 2018. Re-annotation of eight Drosophila genomes. Life Sci. Alliance. 1:1-14. doi: $967 \quad 10.26508 /$ Isa.201800156.

968 Yang Z. 2007. PAML 4: phylogenetic analysis by maximum likelihood. Mol. Biol. Evol. 24:1586969 1591. doi: 10.1093/molbev/msm088.

970 Zhang Y, Sturgill D, Parisi M, Kumar S, Oliver B. 2007. Constraint and turnover in sex-biased gene 971 expression in the genus Drosophila. Nature. 450:233-237. doi: 10.1038/nature06323.

972 Zhao L, Saelao P, Jones CD, Begun DJ. 2014. Origin and spread of de novo genes in Drosophila 973 melanogaster populations. Science. 343(6172):769-72. doi: 10.1126/science.1248286.

974 Zhou Q et al. 2008. On the origin of new genes in Drosophila. Genome Res. 18:1446-1455. doi: 975 10.1101/gr.076588.108.

976 Zile K, Dessimoz C, Wurm Y, Masel J. 2020. Only a single taxonomically restricted gene family in 977 the Drosophila melanogaster subgroup can be identified with high confidence. Genome Biol. 978 Evol. 12(8):1355-1366. doi:10.1093/gbe/evaa127.

\section{Figure and Table Legends}

981 Fig. 1. Venn diagram representing the overlap between the candidate seminal genes we 982 identified (Candidates) and other sets of putative or confirmed D. melanogaster seminal genes. 983 Candidates are those genes we identified (1) to be highly (or differentially) expressed in the 984 accessory glands according to two transcriptomic databases and also (2) to encode secretory 985 proteins with two software programs. Known Seminal Genes (KSGs) are those encoding proteins 986 previously confirmed to be transferred by males into females during mating or those meeting 
stringent multiple criteria that indicate so. Unconfirmed High Confident Candidates (UHCCs) are those Candidates, not included among KSGs, that are both highly and differentially expressed in the accessory glands according to the two consulted transcriptomic databases. Predicted but unconfirmed seminal genes are previously predicted seminal genes not included among KSGs.

Fig. 2. Chromosomal location of $D$. melanogaster seminal genes. Drawings of polytene chromosomes were modified from Lefevre's photographic maps (Lefevre 1976) and gene locations were obtained from FlyBase.

Fig. 3. Mean Ka/Ks $(\omega)$ across the melanogaster group for Known Seminal Genes (KSGs), Unconfirmed High Confident Candidates (UHCCs), and candidate transcription factors driving the expression of seminal genes in the accessory glands (TFs). TFs searches are described in the Identification section and estimation procedures in Methods (Molecular Evolutionary Analyses). The horizontal discontinuous line represents the mean value for all protein-coding genes [according to Haerty et al. (2007)]. Different letters above boxes indicate differences between groups and * indicates differences between the group and the mean value (GLM followed by Tukey comparisons; $p<0.05$ ).

Fig. 4. Seminal genes shared between the melanogaster subgroup and other Diptera. Numbers refer to the 196 Drosophila orthogroups (generated with Orthofinder) having at least one seminal gene of the melanogaster subgroup. Orthogroups having seminal genes of various taxa are represented by overlapped areas.

Fig. 5. Duplication (blue), loss (magenta), and de novo emergence (black) events among orthogroups containing $D$. melanogaster seminal genes. The numbers of events are shown per branch. Since orthogroups without $D$. melanogaster SFPs were not considered, de novo gains for branches outside the $D$. melanogaster lineage, which are zero, are not shown. Divergence times were obtained from Obbard et al. (2012).

Fig. 6. Expected gene family topology for each considered mechanism of seminal gene origin. Ingroup genes represent melanogaster genes, while outgroup genes represent genes of any nonmelanogaster group species for which seminal genes are known. Magenta branches correspond to seminal genes, while black branches correspond to non-seminal genes. Grey discontinuous branches stand for the absent of homologs.

Fig. 7. Most likely mechanisms of origin of $D$. melanogaster seminal genes. Mechanisms were proposed according to our analysis of seminal gene families only for tentatively young seminal genes, i.e., those that have likely emerged after the split from the lineage leading to $D$. pseudobscura. Uncertain genes represent those we could not determine whether they are young or ancient. 
1021 Table 1. List of Unconfirmed High Confident Candidates (UHCCs). Name, chromosomal location, 1022 and molecular function (taken from FlyBase r2020_03) are shown for each gene.

1023 Table 2. Molecular function annotation of Known Seminal Genes (KSGs) and Unconfirmed High 1024 Confident Candidates (UHCCS). For each group, count (and percentage) and false discovery rate 1025 (FDR) are shown for each GO term found with DAVID with more than one gene.

1026 Table 3. D. melanogaster seminal transcription factors candidates. Aligment e-value and the 1027 assigned DNA-binding domain family are shown for each candidate found with AnimalTFDB3.0. 1028 The first search was performed on genes whose expression strongly correlates to KSGs expression 1029 according to Ayroles et al. (2011). The second search was performed on genes whose expression 1030 is enriched in the male accessory glands according to modENCODE and FlyAtlas 2 D. virilis search, 1031 which was performed using Blastp (alignment bit score $>80$ ), shows the presence/absence of 1032 homologs among the $D$. virilis putative seminal TFs. 


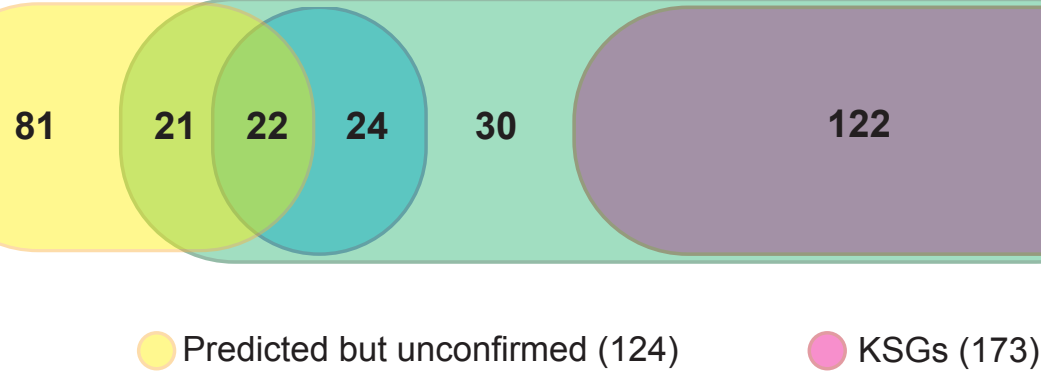




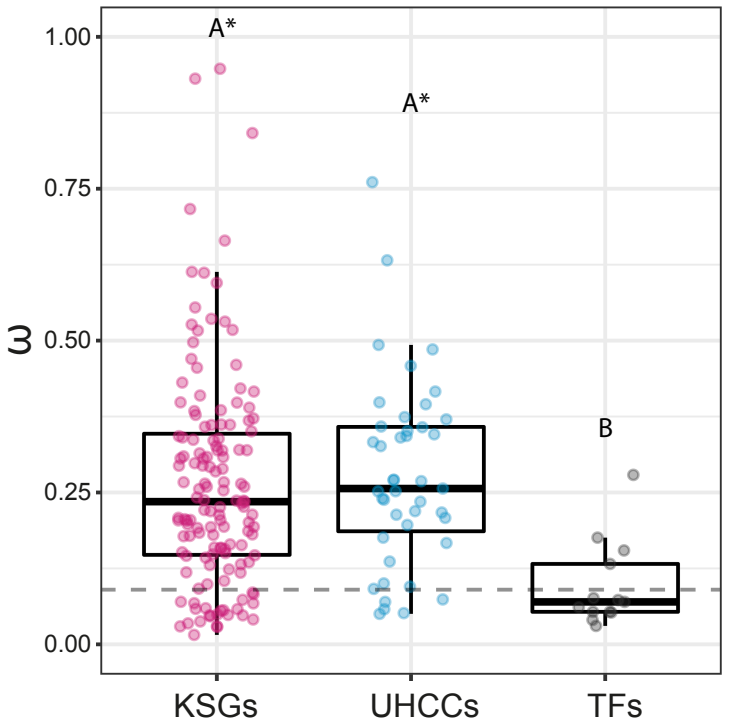



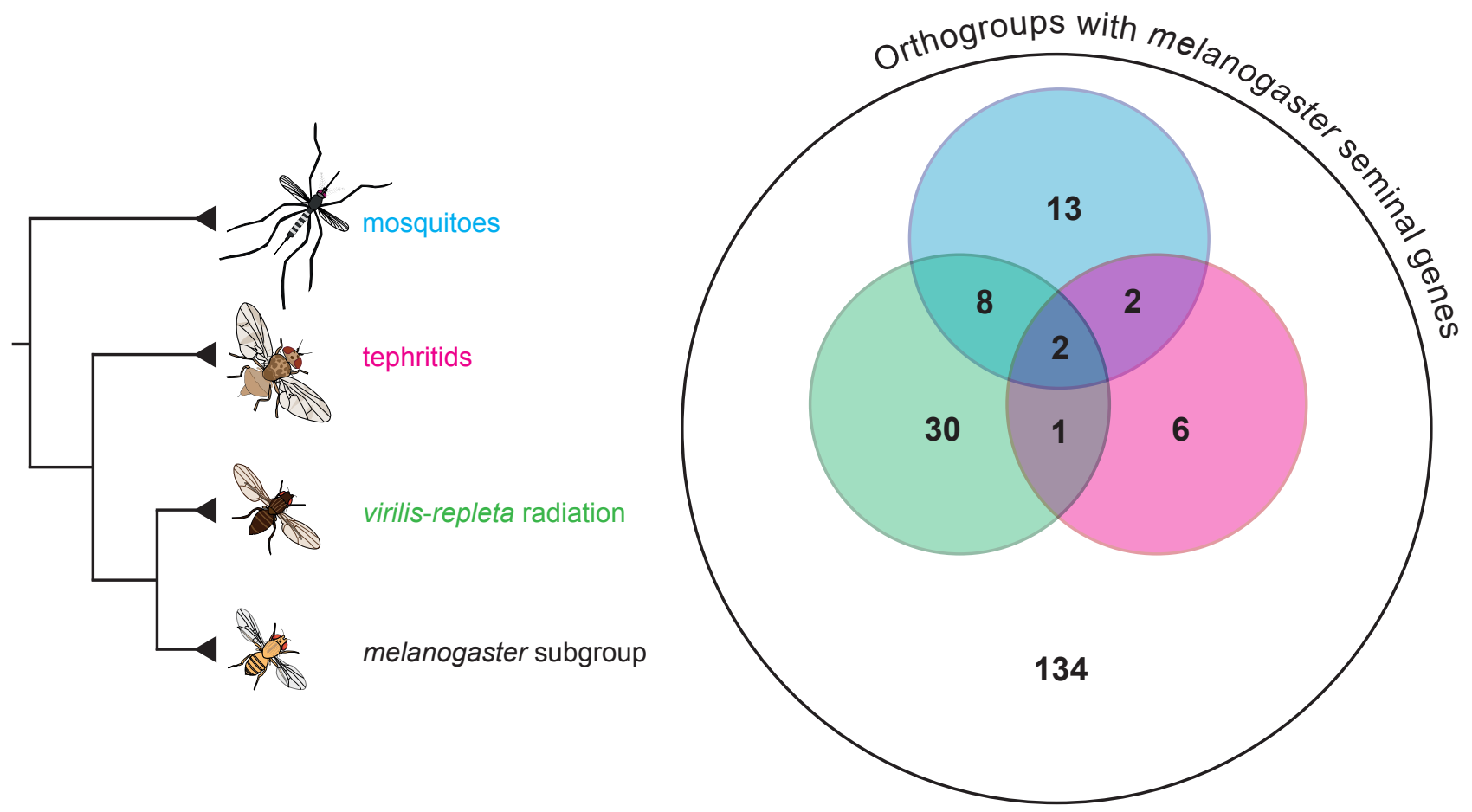

also containing (homologs to) seminal genes of: mosquitoes virilis-repleta radiation tephritids 


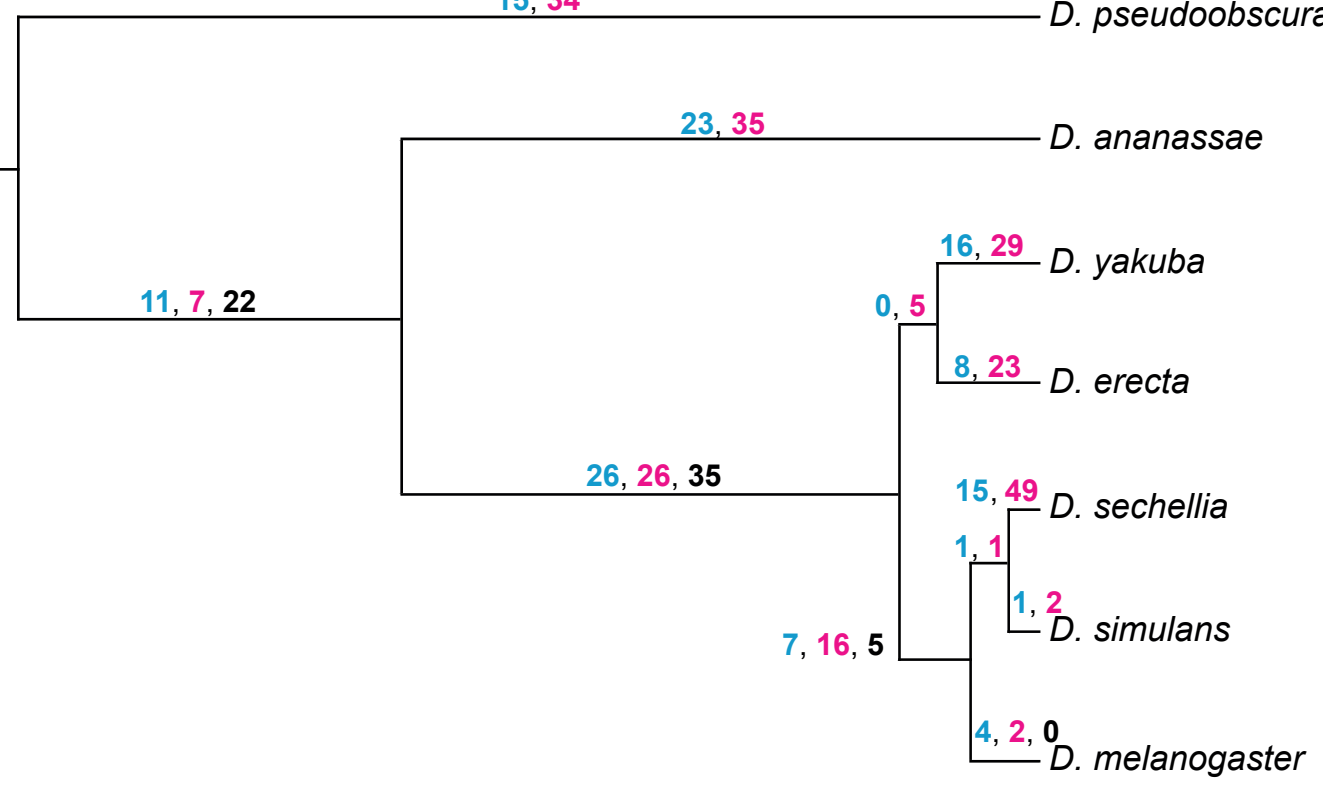


Duplication

outgroup seminal gene

Co-option without duplication

outgroup non-seminal gene

ingroup seminal gene
Duplication-mediated co-option

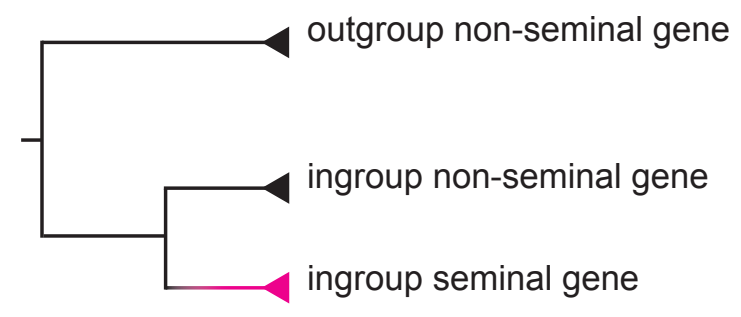

De novo emergence

ingroup seminal gene 


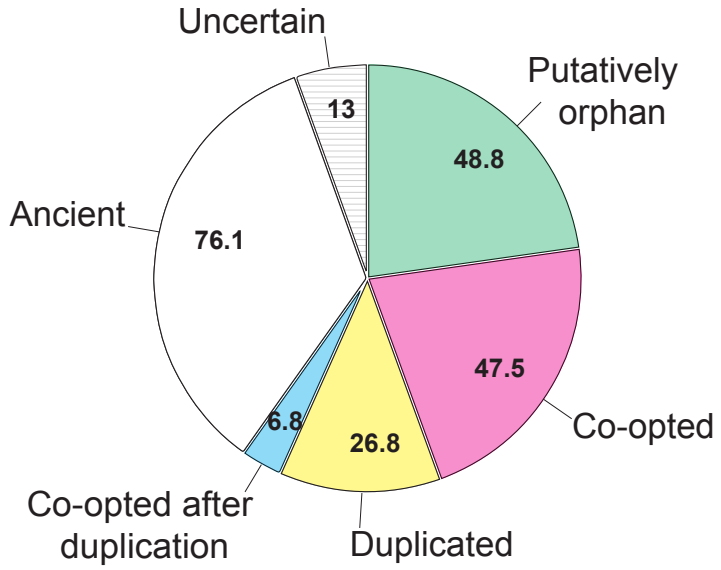




\begin{tabular}{|c|c|c|c|c|}
\hline $\begin{array}{l}\text { Name or } \\
\text { symbol }\end{array}$ & FlyBase ID & $\begin{array}{c}\text { Novel } \\
\text { candidate }\end{array}$ & $\begin{array}{l}\text { Chromosomal } \\
\text { location }\end{array}$ & $\begin{array}{l}\text { Molecular function } \\
\text { (GO) }\end{array}$ \\
\hline Manf & FBgn0027095 & Yes & $3 R$ & unknown \\
\hline CG4271 & FBgn0031409 & Yes & $2 \mathrm{~L}$ & serine-type endopeptidase/hydrolase activity \\
\hline atilla & FBgn0032422 & Yes & $2 \mathrm{~L}$ & unknown \\
\hline CG17549 & FBgn0032774 & No & $2 L$ & unknown \\
\hline CG9336 & FBgn0032897 & Yes & $2 \mathrm{~L}$ & unknown \\
\hline CG11112 & FBgn0033164 & No & $2 \mathrm{R}$ & unknown \\
\hline CG11113 & FBgn0033165 & No & $2 \mathrm{R}$ & unknown \\
\hline Gbp1 & FBgn0034199 & Yes & $2 \mathrm{R}$ & cytokine activity \\
\hline CG13557 & FBgn0034867 & Yes & $2 \mathrm{R}$ & unknown \\
\hline CG12310 & FBgn0036467 & Yes & $3 L$ & unknown \\
\hline CG11977 & FBgn0037650 & No & $3 R$ & unknown \\
\hline CG8420 & FBgn0037664 & No & $3 R$ & unknown \\
\hline SPH2O2 & FBgn0039599 & No & $3 R$ & serine-type endopeptidase activity \\
\hline Lectin-21Ca & FBgn0040107 & No & $2 \mathrm{~L}$ & carbohydrate binding \\
\hline BG642312 & FBgn0047334 & No & $3 \mathrm{~L}$ & unknown \\
\hline CG31997 & FBgn0051997 & Yes & 4 & unknown \\
\hline CG32382 & FBgn0052382 & No & $3 \mathrm{~L}$ & serine-type endopeptidase/hydrolase activity \\
\hline CG33290 & FBgn0053290 & No & $3 \mathrm{~L}$ & unknown \\
\hline Acp54A1 & FBgn0083936 & No & $2 \mathrm{R}$ & unknown \\
\hline CG34299 & FBgn0085328 & Yes & $3 R$ & unknown \\
\hline CG34103 & FBgn0250831 & No & $3 R$ & unknown \\
\hline CG15394 & FBgn0250835 & No & $2 \mathrm{~L}$ & unknown \\
\hline CG42471 & FBgn0259961 & No & $2 \mathrm{~L}$ & unknown \\
\hline CG42481 & FBgn0259971 & Yes & $3 L$ & unknown \\
\hline CG42521 & FBgn0260396 & Yes & $3 L$ & unknown \\
\hline CG12163 & FBgn0260462 & Yes & $3 R$ & cysteine-type peptidase/hydrolase activity \\
\hline CG42852 & FBgn0262099 & Yes & $3 L$ & unknown \\
\hline CG43057 & FBgn0262359 & No & $2 \mathrm{~L}$ & unknown \\
\hline CG43061 & FBgn0262363 & No & $3 R$ & unknown \\
\hline CG43101 & FBgn0262547 & No & $2 \mathrm{R}$ & unknown \\
\hline CG43123 & FBgn0262583 & No & $2 \mathrm{R}$ & unknown \\
\hline CG43185 & FBgn0262814 & Yes & $2 \mathrm{~L}$ & unknown \\
\hline CG43254 & FBgn0262899 & Yes & $3 R$ & unknown \\
\hline CG43267 & FBgn0262948 & Yes & $2 \mathrm{R}$ & unknown \\
\hline CG43350 & FBgn0263082 & Yes & $2 \mathrm{~L}$ & serine-type endopeptidase inhibitor activity \\
\hline CG43392 & FBgn0263249 & Yes & $3 \mathrm{~L}$ & unknown \\
\hline CG43679 & FBgn0263762 & Yes & $3 L$ & unknown \\
\hline CG43788 & FBgn0264329 & Yes & $2 \mathrm{R}$ & unknown \\
\hline CG43789 & FBgn0264330 & Yes & $2 \mathrm{R}$ & unknown \\
\hline CG44102 & FBgn0264911 & Yes & $2 \mathrm{R}$ & unknown \\
\hline CG13639 & FBgn0265266 & No & $3 R$ & unknown \\
\hline CG18258 & FBgn0265267 & No & $x$ & carboxylic ester hydrolase activity \\
\hline CG44388 & FBgn0265538 & Yes & $2 \mathrm{R}$ & unknown \\
\hline
\end{tabular}


bioRxiv preprint doi: https://doi.org/10.1101/2021.05.11.443674; this version posted May 12, 2021. The copyright holder for this preprint (which was not certified by peer review) is the author/funder. All rights reserved. No reuse allowed without permission.

$\begin{array}{lllll}\text { CG44574 } & \text { FBgn0265785 No } & \text { 2L } & \text { unknown } \\ \text { CG45011 } & \text { FBgn0266363 No } & \text { 2L } & \text { unknown } \\ \text { CG45012 } & \text { FBgn0266364 Yes } & \text { 2L } & \text { unknown }\end{array}$




\begin{tabular}{|c|c|c|c|c|}
\hline \multirow{2}{*}{ GO term } & \multicolumn{2}{|c|}{ KSGs } & \multicolumn{2}{|c|}{ UHCCS } \\
\hline & Count & FDR & Count & FDR \\
\hline serine-type endopeptidase inhibitor activity & $18(10.4 \%)$ & $1.76 \mathrm{E}-16$ & 0 & - \\
\hline hormone activity & $6(3.5 \%)$ & $3.09 E-04$ & 0 & - \\
\hline galactose binding & $5(2.9 \%)$ & $3.09 E-04$ & 0 & - \\
\hline lipase activity & $5(2.9 \%)$ & 0.00414 & 0 & - \\
\hline serine-type endopeptidase activity & $11(6.4 \%)$ & 0.00804 & $3(6.5 \%)$ & 0.07748 \\
\hline odorant binding & $7(4.0 \%)$ & 0.01045 & 0 & - \\
\hline flavin-linked sulfhydryl oxidase activity & $3(1.7 \%)$ & 0.01045 & 0 & - \\
\hline peptidase inhibitor activity & $3(1.7 \%)$ & 0.01362 & 0 & - \\
\hline carbohydrate binding & $6(3.5 \%)$ & 0.01389 & 0 & - \\
\hline \multirow{2}{*}{ hydrolase activity } & $4(2.3 \%)$ & 0.02990 & \multirow{2}{*}{$3(6.5 \%)$} & \multirow{2}{*}{0.07739} \\
\hline & $4(2.3 \%)$ & 0.19229 & & \\
\hline protein disulfide isomerase activity & $3(1.7 \%)$ & 0.09327 & 0 & - \\
\hline thiol oxidase activity & $2(1.2 \%)$ & 0.18233 & 0 & - \\
\hline unannotated & $77(44.5 \%)$ & - & $37(80.4 \%)$ & - \\
\hline
\end{tabular}




\begin{tabular}{|c|c|c|c|c|c|c|}
\hline $\begin{array}{c}\text { Name or } \\
\text { symbol }\end{array}$ & FlyBase ID & TF family & e-value & $\begin{array}{c}\text { First } \\
\text { search }\end{array}$ & $\begin{array}{l}\text { Second } \\
\text { search }\end{array}$ & $\begin{array}{c}\text { D. virilis } \\
\text { search }\end{array}$ \\
\hline retn & FBgn0004795 & ARID & $3.10 \mathrm{E}-22$ & Yes & No & No \\
\hline CG7556 & FBgn0030990 & MYB & $5.00 \mathrm{E}-16$ & Yes & Yes & No \\
\hline prd & FBgn0003145 & PAX & $1.10 \mathrm{E}-71$ & Yes & Yes & Yes \\
\hline toe & FBgn0036285 & PAX & $1.00 \mathrm{E}-33$ & Yes & Yes & Yes \\
\hline CG13559 & FBgn0034870 & zf-LITAF-like & 5.30E-17 & Yes & Yes & No \\
\hline CG6470 & FBgn0030933 & $\mathrm{zf}-\mathrm{C} 2 \mathrm{H} 2$ & 0.00020 & Yes & Yes & No \\
\hline CG17841 & FBgn0028480 & TRAM_LAG1_CLN8 & $2.60 E-63$ & Yes & No & No \\
\hline Myс & FBgn0262656 & bHLH & $5.90 \mathrm{E}-11$ & Yes & No & Yes \\
\hline CrebA & FBgn0004396 & TF_bZIP & $3.20 \mathrm{E}-15$ & No & Yes & Yes \\
\hline stc & FBgn0001978 & zf-NF-X1 & $1.10 \mathrm{E}-10$ & No & Yes & Yes \\
\hline CG3065 & FBgn0034946 & $\mathrm{zf}-\mathrm{H} 2 \mathrm{C} 2 \_2$ & $4.60 \mathrm{E}-22$ & No & Yes & Yes \\
\hline Bap111 & FBgn0030093 & HMG & $1.30 \mathrm{E}-16$ & No & Yes & No \\
\hline pzg & FBgn0259785 & $\mathrm{zf}-\mathrm{C} 2 \mathrm{H} 2$ & 7.50E-09 & No & Yes & Yes \\
\hline CG11414 & FBgn0035024 & $\mathrm{zf}-\mathrm{C} 2 \mathrm{H} 2$ & 7.00E-05 & No & Yes & No \\
\hline
\end{tabular}

